SCHOOL-AGED CHILDREN'S AWARENESS

OF THEIR OWN WORKING MEMORY CONTENTS

\begin{tabular}{c} 
A Dissertation \\
presented to \\
the Faculty of the Graduate School \\
at the University of Missouri-Columbia \\
In Partial Fulfillment \\
of the Requirements for the Degree \\
Doctor of Philosophy \\
\hline Dr. Nelson Cowan, Dissertation Supervisor \\
DECEMBER 2018 \\
bH by
\end{tabular}


The undersigned, appointed by the dean of the Graduate School, have examined the dissertation entitled

\section{SCHOOL-AGED CHILDREN'S AWARENESS OF THEIR OWN WORKING MEMORY CONTENTS}

presented by Christopher L. Blume

a candidate for the degree of doctor of philosophy, and hereby certify that, in their opinion, it is worthy of acceptance.

Professor Nelson Cowan

Professor Judith Goodman

Professor Kristy van Marle

Professor Steve Hackley 


\section{ACKNOWLEDGEMENTS}

I would like to thank Professor Nelson Cowan for his many years of guidance beginning with my time in the Working Memory lab as an undergraduate research assistant and continuing beyond my dissertation defense. I would also like to thank Professor Steve Hackley for his instruction of the historical perspectives throughout the history of psychological research. Additional thanks go to Professor Kristy van Marle for providing a critical developmental perspective outside of my own cognitive perspective and to Professor Judith Goodman for providing the outside perspective so instrumental in discussing the topics of psychology to one whose expertise lies elsewhere in research. Finally, I would like to thank Bret Glass for his assistance with recruiting the child participants necessary for this project. 


\section{TABLE OF CONTENTS}

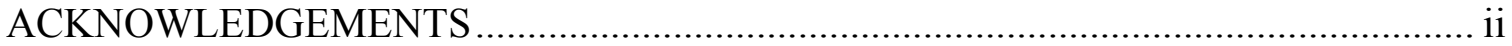

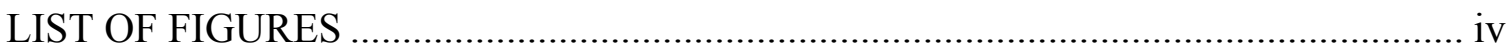

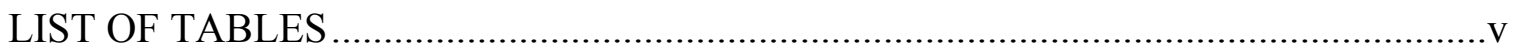

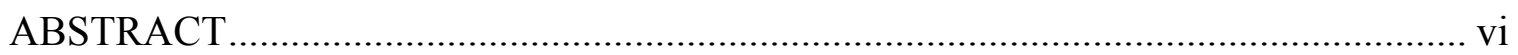

Chapter

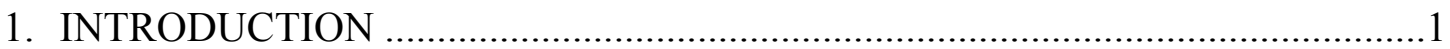

Research on Working Memory and its Development

Research on Metamemory in Relation to Working Memory

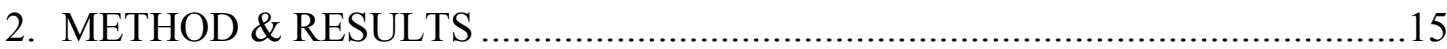

Visual Array Task Overview

Auxiliary Task Overview

Experiment 1

Experiment 2

Experiment 3

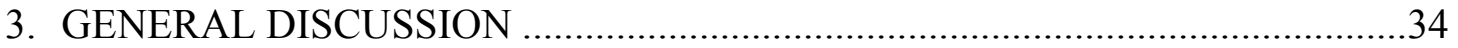

APPENDIX

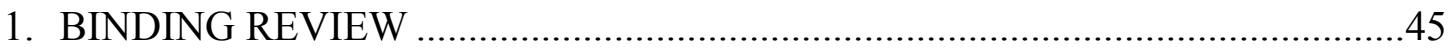

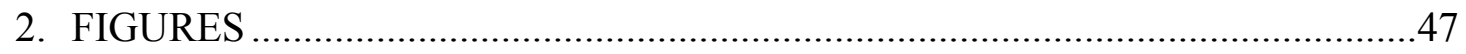

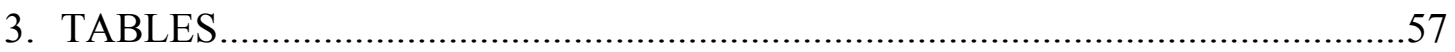

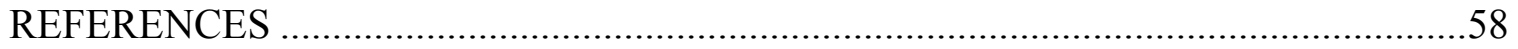

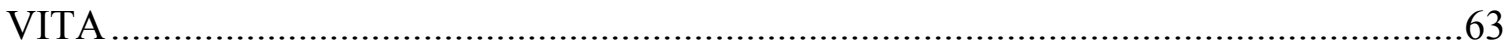




\section{LIST OF FIGURES}

Figure

1. Design for Experiment 1 without (1a) and with (1b) metamemory storage judgment.

2. Experiment 1 mean estimates of capacity $(\mathrm{k})$ and storage judgments (Meta) .........48

3. Experiment 1 scatterplot of participants' mean capacity $(\mathrm{k})$ and mean storage judgment

4. Experiment 1 response times (RT) for providing a verbal storage judgment and for responding to the probe as Same or Different

5. Design for Experiment 2 conditions without (left panel) and with (right panel)

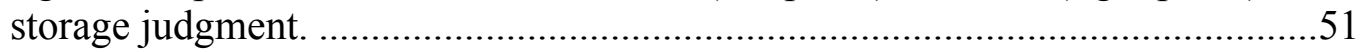

6. Experiment 2 mean capacity $(\mathrm{k})$ estimates by group and condition .......................52

7. Experiment 2 scatterplot of participants' mean capacity $(\mathrm{k})$ and mean storage judgment.

8. Experiment 2 response times (RT) for providing a verbal storage judgment and for responding to the probe as Same or Different.

9. Experiment 3 mean results for metamemory Storage Judgments (with SEM) by Age Group, Set Size (SS), and Retention Interval (RI).

10. Experiment 3 mean results for Mean $k$ estimates (with SEM) by Age Group, Set Size (SS), and Retention Interval (RI) 


\section{LIST OF TABLES}

$\begin{array}{lll}\text { Table Page } & \end{array}$

1. Experiment 1 measurement means (with SEM) by age Group ................................57 


\title{
SCHOOL-AGED CHILDREN'S AWARENESS \\ OF THEIR OWN WORKING MEMORY CONTENTS
}

\author{
Christopher L. Blume \\ Dr. Nelson Cowan, Dissertation Supervisor
}

\begin{abstract}
Working memory researchers in psychology have long wondered about how the mind organizes the many different pieces of information that must be maintained at any one time in order that the individual may carry out daily tasks of cognition. This research has often focused on the capacity of information that an individual is capable of holding in mind at any one time. In order to obtain a better understanding of this capacity researchers have developed what are thought to be objective measures of estimating the number of items $(k)$ an individual must have in mind based on their performance on some cognitive task. In the present research one such formula is used to obtain a typical estimate for a visual array task in which multiple colored squares must be held in mind for a short duration before the participant is asked about whether or not a single probe color was one of the colors that had just seen in the array.

In addition, participants are asked to provide their own subjective estimates of the number of colors they believe themselves to have memorized. Several age groups were tested starting with children as young as 6 . The results show that while all age groups appear to overestimate their own capacity when compared to the objective $k$ estimate, younger children tend to do so to a greater degree. This effect is discussed as the result of the development of quicker processing with age, faster forgetting in young age, or simply a structural increase in the capacity irrespective of the prior two possibilities.
\end{abstract}




\section{Introduction}

In this project I examined if young children aged 6 to 13 are aware of their own working memory capabilities to the same degree as adults. If they are not as aware, the current study attempts to determine if this can assist in explaining the observation that working memory capacity increases with development. Working memory refers to the small amount of information that can be maintained at once. But this simple maintenance isn't so simple when utilized in the real world. Instead of simple maintenance, working memory includes the ability to switch between different tasks (e.g., switching attention between taking down class notes and listening to the lecturer). The observed ability of humans to switch between tasks indicates that they must be able to build layers of awareness of their own mental contents (i.e., the information currently in working memory) such that they are capable of prioritizing information. Attention is integral to working memory. However, it is generally conceded that not all working memory systems are created equally. Individual differences contribute to performance on working memory tasks including skill level for the ability to switch between tasks, or to stay on task. Kane and McVay (2012), for example, showed that individuals with a lower working memory capacity tended to mind-wander more if a cognitive task was particularly difficult. Interestingly, Levinson, Smallwood, and Davidson (2012) showed a similar effect in the opposing direction. Here, participants with a higher working memory capacity showed more mind wandering if a task was particularly easy. These effects support the idea that working memory necessarily includes monitoring of information that goes in and out of the focus of attention. In the above examples, participants are apparently at least relatively aware of their ability as well as the difficulty level of the 
task at hand. Those lower capacity participants' monitoring tells them the task is beyond their ability and so they don't allocate all of their attention to the task; whereas those higher capacity participants similarly recognize that a simple cognitive task does not require all of their attention. In both of these cases, the participants' awareness of the own ability at least partially dictates their attention. One example of this monitoring process, the notion of refreshing, makes the claim that this monitoring of lower-level awareness is achieved (at least partially) by continually cycling the secondary information into awareness before very quickly attending back to the primary information relevant for task completion. You can plug this idea into a cognitive task such as an Operation Span task in which a person must memorize a list of letters provided one at a time in between solving simple math problems. Individuals completing this task must "kick out" the letters from their primary attention to a background level such as an activated portion of long term memory.

This study investigates possible child developmental changes in the ability of individuals to monitor their own working memory contents (metamemory for working memory information), and whether this ability to monitor accurately plays a role in working memory performance. From this work, in which we collect judgments of how many items an individual thinks are retained in working memory during a trial (storage judgments), we may also gain insight into the processes that contribute to metamemory decisions, such as (1) whether children use external cues in a way that influences the metamemory judgments, and (2) whether overestimates of capacity in metamemory judgments might actually reflect information that was truly present for the metamemory 
judgment but was not in a form that could be used for memory performance, perhaps because of interference from other stimuli.

An alternative possibility is a confounding influence from participants' general tendency to engage in 'middle ground' responding. The previous experiments show that many participants are accurate in estimating the theoretical limit (about three items; Cowan, 2001) of their own working memory. However, it remains possible that participants are merely likely to claim the middle option. This phenomenon has been observed for responses to Likert scale questionnaires (Klopfer \& Madden, 1980) as well as the related time-order error (TOE; Hellström, 1985). TOE is the effect in which people tend to judge similar stimuli as of different magnitude when separated in time (e.g., judging two identical sounds heard before and after an inter stimulus interval (ISI) as of different volumes). The TOE has been suggested as related to duration since initial stimulus presentation because participants "are required to compare...objects about which they possess different amounts of information” (p. 57, Hellström, 1985). Evidence for middle ground responding therefore can include a general tendency to provide a storage judgment halfway between zero and the full array, or seeing an effect in which middle ground responding is only present for the longer RI. Previous research has shown that individuals will at times tend toward some middle response, especially as the RI increases. (Cowan \& Morse, 1986; Hellström, 1985). For example, two auditory stimuli of the same pitch are judged as more different as the RI increases because the first pitch is retroactively judged as more intermediate. Judgments, in this case, are more accurate initially but become more intermediate as information about an item in memory is lost. Each of these phenomena, and potentially the present work, pertain to the error of central 
tendency (Hollingworth, 1910), or what I have referred to as middle ground responding. Experiment 3 will test for this confound before examination of more theoretical explanations described in more detail below.

I have designed a series of experiments meant to specifically determine if children are able to accurately estimate their own working memory contents. In this way I hope to gain insight into what particular mental skill is lacking in children causing their deficient working memory ability compared to adults. Knowledge about the information contents of one's own memory may provide additional retrieval power as well as an enhanced ability to correct mistakes. Awareness of your own memory requires that the memories be attended in highly specific detail. This can include knowing the source of the information, knowing the order of information, knowing the purpose of the information, etc. Meanwhile, error correction necessarily requires that you are aware of when a mistake is made. In the current estimation-of-mental-contents metamemory task this awareness means knowing when you have over or underestimated your own ability. A child who is unaware of their own poor memory may be less likely to specifically attempt to make improvements to their own encoding and consolidation of memory. It has been shown through event-related potential (ERP) experiments that individuals are aware of when a mistake has occurred (Holroyd \& Coles, 2002; Yeung, Botvinick, \& Cohen, 2004). The participants in the present experiments are provided additional information about how errors can occur (e.g., they may notice that their error rate increases on certain less attended trials or recognize that errors increase when they make higher capacity estimates). 
In the present study it is proposed that younger children have difficulty estimating the number of visual items in mind because of difficulty in retention of memoranda until a memory test can be administered. In the following sections I will (1) provide a review of pertinent literature for working memory capacity and its development, and then (2) of participants' metamemory or awareness of their capacity and its development; and (3) in the methods section, describe the basic visual array change-detection task used in the current experiments and (4) the metamemory assessment addition to the visual array task aimed at examining awareness of one's personal working memory contents.

\section{Research on Working Memory and its Development}

Issues in working memory. Working memory, or the process of maintaining a

small amount of information for immediate use, has become a popular topic over the last two decades. Working memory is such a widely utilized mental capacity that it has become of great interest to study it from every different angle imaginable. Theories abound. They assert contradictory properties of working memory, such as whether or not the information is being maintained as discrete items or as a continuous resource (Bae \& Flombaum, 2013; Bays \& Husain, 2008; Thiele, Pratte, \& Rouder, 2011; Zhang \& Luck, 2008). The answer to this question could then inform further research topics such as feature binding, e.g., memorizing not just that you saw a red shape or a square, but you mentally bind the two features into a red square (Cowan, Blume, \& Saults, 2013; Hardman \& Cowan, 2015; Oberauer \& Eichenberger, 2013), and how information can be manipulated if different modalities are separately maintaining the items, i.e., how does feature binding occur if the features are stored separately (Fougnie \& Marois, 2011; 
Saults \& Cowan, 2007; Cowan, Saults, \& Blume, 2014; Vergauwe, Barrouillet, \& Camos, 2010).

Individual differences in working memory. Individual differences within working memory have also been examined many times as researchers have found that not everyone's working memory is the same (Gulbinaite, Johnson, de Jong, Morey, \& van Rijn, 2015; Unsworth, Fukuda, Awh, \& Vogel, 2014). The potential group and individual differences within working memory suggest that knowledge of the developmental trajectory of working memory could assist in forming the best possible picture of how the process works. There remains the potentiality that so many competing theories on working memory processes can't seem to be clearly decided because not all individuals' working memory systems are the same.

Developmental differences in working memory. Individual differences among adults are not the only way in which working memory systems can importantly differ between individuals. They also may play a role in determining the starting point for an individual's working memory development. Developmental differences may include not only capacity differences, but entirely different organizations of working memory (Cowan \& Alloway, 1997). Gathercole, Pickering, Ambridge, and Wearing (2004) suggest that working memory is structurally formed by age six (the youngest age of participants in the current study), but maintain that functional capacities continue to expand through adolescence. In other words, working memory is structurally complete by age 6 , but capacities for the suggested modular structures in working memory continue increasing for many more years of development. For example, a person with a lower working memory capacity, who is aware of this fact, may need to more heavily rely on 
'work arounds' than a person with a higher working memory capacity. That children do so is by no means a known fact, though it does shed light on just how important it is for teachers or parents to be informed of better, evidence-based knowledge about the development of the working memory of children just beginning their education. Different developmental trajectories may develop leading to multiple viable 'final' organizations of adult working memory. The development of working memory has not gone ignored. Working memory has been studied throughout the lifespan from infants and young children (Courage \& Cowan, 2008), through adolescence and young adulthood, and on into more advanced ages (Salthouse, 2015).

Each of these major topics of interest can be considered as an investigation of the capacity of working memory. The capacity of working memory could prove essential knowledge for designing individual curricula based on a child's cognitive abilities. One highly relevant example is the connection between working memory capacity and reading skill. Reading skills correlate with measures of working memory and intelligence (Daneman \& Carpenter, 1980). While it remains to be seen whether working memory skills can be improved through practice (see Melby-Lervåg, Redick, \& Hulme, 2016), attempts have already been made (with some success) to incorporate working memory training into schools (Holmes \& Gathercole, 2014). Whether the question is how many items can be held, by what mechanisms are the items held, or how to improve our capacity; our research questions give the indication that we want to know everything about working memory's limits.

Diverse fields of study maintain a substantial interest in working memory thanks largely due to how useful a tool it is in relation to a number of other mental 
characteristics. One of the most widely studied phenomena is the relationship between intelligence (i.e., general fluid intelligence, or $g$ ) and working memory span. Intelligence presents a problem in understanding because there is no single way to describe it. It could refer to knowledge of semantic facts, mental speed in solving a common problem, creative thinking ability to solve a unique problem, or simply math ability (XenidouDervou, de Smedt, van der Schoot, \& van Lieshout, 2013). Intelligence and working memory show a monotonic relationship such that as one increases with development, the other does likewise at a similar pace. The cognitive development of working memory and intelligence, therefore presents a crucial test of the relationship. In general, it appears that a cognitive standing within an age cohort remains stable throughout childhood through adulthood. In other words, a 12 year old in the top $10 \%$ of her cohort in terms of working memory span and intelligence is unlikely to drop to the lower half by age 42 .

The cognitive development of working memory ability presents the opportunity to examine in what ways working memory changes, improves, and deteriorates. It is important to understand the reason behind working memory development and the possible role of metamemory for school-aged children in particular. School children are often advanced through a curriculum at an identical pace and manner of teaching without individual adjustments. It may be that some, if not many, of these children would greatly benefit from an educational strategy that takes into account each child's cognitive abilities and differences. A child who starts off at a disadvantage may remain at the disadvantage throughout the remainder of her school years because the curriculum assumes a certain knowledge base inasmuch as the child passed the preceding grade levels. The most extreme case of this issue is the possibility that the set curriculum is not 
ideal for any students. It may simply 'work well enough' to have not elicited change. A better understanding of how much information children can handle, how quickly they can handle that information, and how accurate they are when they describe their own performance levels may assist in making a preliminary determination as to whether specialized assistance based on skill level is warranted.

The adults in our study include groups of college undergraduates who have only recently finished their primary education, as well as middle-aged adults who have long since finished their education. Children are known to have a worse working memory than adults, with a level of performance that increases with age in childhood, but why this is the case is not known. The mental mechanics for working memory performance is important to know because working memory is important for school performance (Cowan, 2014).

The current study models the basic change-detection format of Luck and Vogel (1997) with the addition of a metamemory task following that of Cowan, Hardman, Saults, Blume, Clark, and Sunday (2016). This common visual array task (Figure 1a) presents a participant with five colored squared for $500 \mathrm{~ms}$ followed by a query of how many of the five items the participant believes herself to have successfully attended for a later memory test. The memory test immediately follows the storage judgment allowing for the possibility to ascertain each participant's mean $k$ score along with a personal introspective judgment on what each participant believes her own $k$ to be. Many different avenues of inquiry have built an impressive framework for our knowledge of working memory as a general process. Here we investigate the individual personal ability to 
recognize, or be aware of how well our own working memory is performing from our earliest days in formal education through our middle adult life.

\section{Research on Metamemory in Relation to Working Memory}

Metamemory refers to direct knowledge of one's own memory. Knowledge or awareness of cognitive ability is important in order to use one's knowledge most effectively. I will explore working memory capability in relation to metaknowledge of working memory contents, or metamemory for this information.

In the present study, we determine if our awareness of our own working memory capabilities increases along with the capabilities themselves (i.e., does higher working memory capacity in more capable or older participants relate to higher metamnemonic capacity judgments). This awareness may be the results of one, or several processes. The experiments here will investigate a few possibilities. Specifically, the basic design (Figure 1b) outlined below (and in more detail preceding each Experiment) will be used to test the possibility that children's worse performance is the result of (1) a lower capacity of working memory, (2) slower processing of working memory, or (3) quicker forgetting of working memory. Testing the individuals' awareness of their own memory may be conducive to the individual asking these questions of themselves (e.g., "I know that I had all the items at first, but by the time I was tested I forgot some.") as well as allowing us to interpret how they seem to have responded to these questions internally. In the example introspection above the participant may respond by lowering the number of items they even attempt to memorize or by sticking to their overestimation claim and instead trying to focus more on the maintenance of the items. Cowan et al. (2016) provide an option for measuring introspection other than confidence rating in the form of storage 
judgments. The storage judgment method asks participants to identify how many of the items in an array they believe they were able to encode into working memory, whereas the confidence rating method asks only about your belief in how well you already responded. The latter method could then be considered a measure of awareness of performance, whereas the former is more accurately described as a measure of awareness of mental contents at any one moment. These issues will be generally addressed by using different array set sizes different allotted time to process the arrays, different auxiliary tasks, and a test on memory awareness of the array.

Confidence ratings and remember/know. Metamemory research appears to have been largely dominated by the method of collecting confidence ratings of assertions of memory performance. Wilken and Ma (2004), for example, collected confidence ratings on a change detection task similar to those previously described. Each trial in this study presented an array of colors followed by a second array that could have changed anywhere from none to all of the array items. Confidence ratings here can show that participants became more confident in their ability to detect changes as the total number of changes increased. However, participants were only required to respond as to whether a change of any kind had occurred, therefore it is to be expected that confidence increases as the potential number of changes detected increases. Rademaker, Tredway, and Tong (2012) showed, in addition, that greater confidence ratings correspond to more precise memory performance. This provides some evidence that we are aware of our relative memory capability.

However, confidence ratings such as these are flawed for two important reasons. First, such ratings are entirely subjective and could therefore fail to provide truly useful 
information about an individual's working memory capacity. Confidence ratings can be helpful in deciphering what information a particular person actually remembers compared to well known information (i.e., confidence ratings can assist generating theory for remember/know tasks; Wixted \& Stretch, 2004). A second flaw in the confidence rating metamemory studies presented here is that the confidence rating follows the memory probe task. Confidence ratings are an inherently subjective and retroactive task. The act of responding to a particular probe will make confidence judgments even more subjective as they will now incorporate direct knowledge of how well a task was completed. In this way confidence ratings following completion of a task trial are a reflection of confidence that a particular response was correct rather than confidence in one's own memory ability. Confidence ratings abound in long term memory research but have been more sparsely utilized in working memory research.

The remember/know method is likewise geared toward studying long term memory. Any influence from knowledge in working memory presents a potential confounding variable best avoided. We were interested in individual predictive evaluation, therefore making the heavily subjective nature of confidence ratings far from ideal (i.e., asserting memory ability prior to test, rather than after realizing if the task was completed correctly already). Another method of assessing metamemory is to obtain direct judgments of how much of an array an individual claims to remember.

Storage judgments. The storage judgment method then has more predictive power over confidence ratings in that such judgments can be made prior to actually seeing a probe or providing a response. In this way, the judgment made remains unaffected by the immediately preceding memory performance. Cowan et al. (2016) 
presented an array of five colored squares (Exp. 1) identical to that of the present report. However, whereas the current experiment contains a single probe item as is usual for this change detection paradigm, Cowan et al. presented a full probe array asking for an estimation of how many colors have been changed. The full array was used in this case to assist in the formation of several competing models of the data. The present paper represents an attempt to ascertain the ability of younger children to comprehend and complete the metamemory task of storage judgments like those of Cowan et al. and therefore reduces the complexity of the change detection task to the more manageable single change detection.

Young children have been shown to have less total cognitive resources at their disposal than adults (Luciana, Conklin, Hooper, \& Yarger, 2005). We decided it would be prudent to investigate children's ability to comprehend and complete the basic storage judgment task alongside the change detection task rather than discovering the introspection is too difficult for them to accomplish when they must detect multiple changes. Attention has been described as a fluctuating factor for working memory capacity estimation (Rouder et al., 2008) that may lead to an inconsistent trial-by-trial capacity estimation (van den Berg, Awh, \& Ma, 2014). We feel the simplification of the current study compared to Cowan et al. (2016) will provide a framework for investigating development of working memory in several key ways. The total cognitive resources of an individual includes their ability to attend stimuli or, conversely, their proclivity for inattention (Kane \& McVay, 2012). The multiple change detection method could potentially extend beyond the basic cognitive capabilities of younger children in that they may lack the necessary attentive capacity to keep track of several changes as well as 
trying to introspect on the number of memoranda they have in mind (e.g., inhibition may be a cognitive skill that hinders working memory in young children; Borella \& Ribaupierre, 2014). By reducing the possible changes to one we alleviate some of the attention requirement as only a single item needs to be stored for more than a few seconds. Individuals of all ages will be able to make their storage judgments with the foreknowledge that they will be able to drop all but one of the items as soon as the probe appears on screen, rather than maintaining attention on all items even through the probe. 


\section{Method \& Results}

\section{Visual Array Task Overview}

The visual array change-detection task has been extremely popular since Luck and Vogel (1997) first used it. This is perhaps due to its relative simplicity for both participants and researchers. Participants are momentarily shown an array of colors to remember followed by a query about the array. The query is generally presented as a single color that the participant should either recognize from the array or not. The single stimulus, however, can be asking several different specific questions. The most basic form of this task is to present a single centrally located color for the participant to consider as a part of the array, or not part of the array. Even this basic task allows a mean $k$ to be calculated for each participant. Binding in working memory can also be investigated using this change-detection task simply by presenting the color stimulus

probe in one of the array item locations (e.g., Cowan, Blume, \& Saults, 2013). A short review of binding literature can be found in Appendix A.

Another factor that may contribute to overall working memory capacity is knowledge, or awareness of the items in one's own working memory. We were interested in many possibilities for this factor. First, we were interested in knowing whether or not one's own introspective knowledge of their own working memory relates to actual working memory capacity differences.

Does belief represent truth in working memory capability? Additionally, we were interested in how this introspective ability may develop with age. The notion of an increasing working memory capacity with age is well founded in the literature on arraymemory tasks, like many other procedures (e.g., Cowan et al. 2005). We were 
particularly interested in examining if this is reflected in increasing and increasingly accurate judgments of information contained within working memory. In other words, (1) are younger children more inclined to claim a lower working memory than older children and adults, and (2) do introspective claims about the contents of working memory become any more accurate from childhood to adulthood.

\section{Auxiliary Task Overview}

We will also be able to examine the relative fluctuation of individuals based on auxiliary testing on working memory span and intelligence. We expect those participants who score high on auxiliary tests to provide more accurate and less variable storage judgments. Group effects based on age can also be examined despite the simplified method (probing only one item as opposed to the entire array). We expect an improvement in the relatedness of storage judgments and $k$ estimates from younger childhood into adolescence. In other words, as children get older, they will become better at estimating their own memory contents. A second experiment modifies the original task to take into account the possibility that there could be either processing limitations for young children as already discussed, or that the longer retention interval we employ is a hindrance exclusively for younger children. The latter idea follows from work that has postulated at least some of the observed working memory of children compared to adults is due to temporal issues rather than processing limitations (e.g., items decay from memory quicker in children compared to adults; Towse, Hitch, \& Hutton, 1998). The third experiment, as previously stated, will firstly determine if participants simply tend to respond with the middle option, suggesting an error of central tendency (Hollingworth, 1910). 
Overall, we wish to ascertain the degree to which subjective beliefs from introspecting about our own working memory capacity can be said to relate to the objective change detection performance data. The overall purpose of studying these phenomena in school-aged children is meant to provide a preliminary assertion about how much information children can process in working memory, how quickly they can process information in working memory, and how aware they are of their own working memory processing ability. In the following set of experiments we test the possibility that children's worse performance on visual array tasks in working memory is the result of (1) a lower capacity of working memory, (2) slower processing of working memory, or (3) quicker forgetting of working memory.

\section{Experiment 1}

The visual array task has been used on children as young as 6 years old many times in the past and was therefore assumed to be a task that could be reasonably performed by children. However, the storage judgment addition was designed to be performed by adults (Cowan et al. 2016). Experiment 1 therefore was an exploration of the ability of young children to perform this task without making any major changes to the standard visual array design (e.g., timing of the array presentation). Later experiments begin altering the task to examine specific possible explanations for poor performance for children compared to adults.

\section{Participants}

Participants were sampled from five groups. Group 1 was designated for First and Second graders. Group 2 was designated for Third and Fourth graders. Group 3 was designated for Fifth, Sixth, and Seventh graders. Group 4 was designated for young 
adults. Group 5 was designated for middle adults. Respectively for each group there were 29 participants (mean age 7.67 years, 20 female), 24 participants (mean age 9.67 years, 13 female), 29 participants (mean age 11.83 years, 16 female), 28 participants (mean age 20.33 years), and 30 participants (mean age 41.08 years, 23 female). Groups 1-3 were recruited from a database built up from participants from previous research studies as well as new participants reached through a flyer at school, word of mouth from another participant, or an e-mail circulated through the University of Missouri. Group 4 was comprised of undergraduate students participating for course credit. Group 5 is made up of 30 of the parents/guardians who brought in a child for groups 1-3. Groups 1-3 and 5 were compensated with $\$ 5$ per half hour in the experiment. Groups 1-3 received a book in addition to monetary payment. An additional 11 participants were dropped for providing a storage judgment of five on every trial of the change-detection task. This was viewed as failure to understand directions.

\section{Procedure}

All tasks were completed in a sound-attenuated booth with computer-controlled stimuli except for the Ravens progressive matrices, which occurred in a separate testing area using physical stimuli.

\section{Visual Array}

The main experiment was the visual array task with its storage judgment component. The visual array portion of the task presented an array of 5 colored squares to participants for $500 \mathrm{~ms}$. There were 4 practice trials followed by 128 experimental trials. Half of the trials presented a stimulus probe that was from the array just observed while the other half presented a color that was not in the immediately preceding array. A 
change-detection was reported by the participant by pressing one of two buttons on a button box for a color that was the same as one of the five items from the array, or different from all five of them. The same 10 colors were used throughout the experiment selected based on RGB values for easily discriminable colors (black, white, red, blue, green, yellow, brown, cyan, purple, and dark-blue-green). Each of the colored squares were $6 \mathrm{~mm}$ on a side, at least $17.5 \mathrm{~mm}$ from center screen, and at least $17.5 \mathrm{~mm}$ away from the center of any other colored square in the array. All colored squares were drawn on a grey background within an area measuring $74 \mathrm{~mm}$ wide $\mathrm{x} 56 \mathrm{~mm}$ high. The experiment was broken into four parts to allow participants the opportunity to take breaks during the experiment. In addition, each individual trial required a key press to indicate readiness to continue and could have been used as a break point. The stimulus probe was always centrally located and participants could therefore not use any location knowledge to influence their performance.

The storage judgment, or metamemory, portion of the experiment took place in between the array presentation and the probe. Each trial presented a visual mask following the array comprised of nine randomly ordered colors within the location of each of the single-colored array squares for four seconds. A large $(X)$ or (?) was presented along with the visual mask in the center of the computer screen. Trials paired with an $(X)$ required no storage judgment and the participant could merely wait until the stimulus probe appeared. Trials paired with a (?) were an indication that a storage judgment should be made. Participants were instructed that the storage judgment should reflect how many of the items in the array they believe they have in mind. For example, if a participant knows there was a blue square and a black square, but can't recall what the 
other colors were, they should indicate that they know two colors by saying 'two' aloud. The audible response was logged by an experimenter so that the participant could focus their hands solely on the button box to make same/different discriminations.

Each trial began when the participant pressed the SPACE key to begin the trial. A fixation cross was presented center screen for $1000 \mathrm{~ms}$ followed immediately by the array of 5 colored squares for $500 \mathrm{~ms}$. A blank screen then appeared for $500 \mathrm{~ms}$ followed by the mask screen for $4000 \mathrm{~ms}$. Participants were indicated during the mask presentation to either provide a storage judgment (?) or not $(X)$. The storage judgment had to be logged during the mask display or the trial would be scored as incorrect. The single central probe was then presented until a same/different response was made on the button box.

Feedback was provided in order to heighten participant awareness of their performance. Participants will presumably self-correct their metamemory judgments if they do not coincide with performance (e.g., a person who overestimates the number of items in mind may begin to provide smaller judgments following several incorrect responses). The feedback informed participants of their accuracy and reminded them of their visual array (same/different) responses. Additionally, for the metamemory trials the feedback reminded the participant of how many colors they claimed to have had in mind.

\section{Auxiliary Tasks}

Each participant completed an array of tasks including the Ravens progressive matrices, a Running Span task, and a Letter Span task in addition to the main changedetection and storage judgment task. The portion of the Ravens progressive matrices used presented participants with a pattern of images with a section missing. Participants had to select which of the 6 or 8 options should fill in the missing section to complete the 
pattern. Participants in Group 5 did not complete the Ravens portion of the experiment. The Ravens was used to attain some amount of knowledge about each participant's intelligence. The Running Span and Letter Span tasks are each working memory span tasks designed to ascertain the capacity of each participant's worming memory.

The Running Span task (taken from Cowan et al., 2005) acoustically presented 4 numbers per second. Participants were asked to repeat back as many numbers from the end of the list and in the same order they were presented. List length was variable between 12-20 digits and participants were unaware of when each list would cease presenting new numbers. In this way a running mental list of numbers had to be continually maintained and changed. For example, a participant may only be able to maintain four numbers at a time requiring that the oldest number be dropped from mind to be replaced with the newest number. In this way to running span of numbers remains at the capacity of four. These data were scored for accuracy in backward order in order to assess ability to update the numbers in mind. For example, a participant who heard the numbers, “...3, $6,1,7,4,8,2$ " could respond with " $4,8,2$ " for a score of three, or " 7,4 , 8,2 " for a score of four, however, a response such as " $7,4,6,2$ " would result in a score of one as the accuracy scoring ceased as soon as a mistake was made.

The Letter Span task (taken from Lépine, Barrouillet, \& Camos, 2005) presented participants with four letters followed by one number, and repeated up to six times for a total of six numbers presented. A letter was visually presented once per second to be repeated out loud by the participant. After each fourth letter a number would instead be presented for the participant to repeat out loud as well as remembered for later recall. Letters had to be spoken out loud but did not have to be maintained in memory. At the 
pre-determined end of each list the participant was prompted to repeat each of the numbers from the list in the same order they were initially presented. Three trials were conducted at each number level (e.g., 2 number lists, 3 number lists, etc.) and scored based on how many perfect trials were achieved. A perfect trial required that all numbers were recalled in the correct order and each of the distracter letters was spoken aloud.

\section{Results \& Discussion}

Capacity is often considered by using Cowan's (2001) $k$ formula: $N(H-F A)$, or array size multiplied by hits minus false alarms. This working memory capacity (or, $k$ ) estimate works well in a task of some array size in which a single probe item must be cross-referenced with the participant's memory per trial. Mean $k$ estimates of working memory showed a clear developmental increase up to young adulthood. The younger groups each managed to attain a $k$ estimate approximately between 1 and 2 items (Group $1 \mathrm{M}=1.14$ items, $\mathrm{SE}=.11$; Group $2 \mathrm{M}=1.54, \mathrm{SE}=.14$; \& Group $3 \mathrm{M}=2.02, \mathrm{SE}=.12$ ). The adult groups showed greater $k$ capacity than the younger groups (Group $4 \mathrm{M}=2.78$, $\mathrm{SE}=.13 \&$ Group $5 \mathrm{M}=2.90, \mathrm{SE}=.13$ ). Similar developmental patterns were likewise observed for the auxiliary tasks of Running Span (Group $1 M=1.90, S E=.14$; Group $2 M=$ 2.37, $S E=.15$; Group $3 M=2.68, S E=.18$; Group $4 M=2.86, \mathrm{SE}=.12$; \& Group $5 M=3.13$, $S E=.13$ ), Letter $\operatorname{Span}$ (Group $1 M=.62, S E=.11$; Group $2 M=1.18, S E=.13$; Group 3 $M=1.92, S E=.18$; Group $4 M=2.42, S E=.17$; \& Group $5 M=2.61, S E=.17$ ), and Ravens Progressive Matrices (Group $1 M=26.83, S E=1.79$; Group $2 M=33.62, S E=1.93$; Group 3 $M=39.97, S E=1.30$; Group $4 M=\mathrm{N} / \mathrm{A}, S E=\mathrm{N} / \mathrm{A} ; \&$ Group $5 M=49.13, S E=1.17)$. These data are summarized in Table 1. Metamemory and mean $k$ estimates can also be found in Figure 2 . 
An initial question for Experiment 1 was whether or not metamemory storage judgments can be useful in predicting $k$ estimates. A regression was run on mean $k$ estimates with metamemory storage judgments, running span, letter span, and Group as predictors. The regression, $F(4,135)=53.12, p<.0001$, was significant for all four predictor variables including storage judgments, $t(135)=2.47, p<.05$. However, when the regression was run instead on mean storage judgments from participants, $F(4,135)=3.32$, $p<.05$, only the mean $k$ estimates remained significant predictors, $t(135)=2.47, p<.05$. These data provide initial support to the notion of using participants own estimates about their working memory to predict the contents, $k$, of that working memory.

Experiment 1 was also meant to determine if children's estimates of their own working memory contents improved with age. That is to say, do people get better at predicting their own mental capabilities with age? Correlation Matrices support an improving introspective quality for storage judgments particularly between the youngest two groups. Pearson correlations for metamemory storage judgments and mean $k$ estimates are as follows, Group $1 r=.03$, Group $2 r=.19$, Group $3 r=.36$, Group $4 r=.43, \&$ Group $5 r=32$. A scatterplot including trend lines for Experiment 1 correlations can be found in Figure 3.

Finally, Experiment 1 response times (RT) showed that participants of all age groups required about the same amount of time to produce a metamemory storage response (between 2000ms-2300ms). However, RT for making the change detection responses decreased with group age. These RT can be found in Figure 4. Overall, these results suggest that Children were not as skilled at estimating their own working 
memory's as adults. However, there are several possibilities for why this may be the case. These are explored in Experiment 2.

\section{Experiment 2}

Experiment 1 showed that children were able to reliably complete the visual array task with the addition of the storage judgment portion. Experiment 2 therefore begins making changes to the visual array. Experiment 1 additionally showed that Group 1 should be of particular interest. This aligns with the stated purpose of testing young children who are just beginning their school-based education. Experiment 2 therefore tested this group for comparison to an adult group.

\section{Participants}

Participants were sampled from only two groups as the developmental trend observed in Experiment 1 was assumed to carryover for the similar design of Experiment 2. Group 1 was again designated for First and Second graders, whereas Group 2 now consisted of young adults. Groups 1 was recruited and compensated as in Experiment 1 and consisted of 26 individuals (mean age $=7.08$ years, 10 female), and Group 2 consisted of 26 undergraduate students participating for course credit (mean age=19 years, 13 female).

\section{Procedure}

All tasks were completed in a sound-attenuating booth.

\section{Visual Array}

The visual array task used the same colors as Experiment 1 as well as the same size and spacing dimensions. Experiment 2 consisted of four blocks of trials with 32 trials each (Figure 5). Each block began with 2 practice trials. All blocks showed a fixation 
cross for $1000 \mathrm{~ms}$ followed by the visual array of 5 distinct colors for $500 \mathrm{~ms}$. Block $2 \mathrm{~A}$ then presented a $500 \mathrm{~ms}$ blank screen followed by a $500 \mathrm{~ms}$ mask screen of multicolored squares and another $500 \mathrm{~ms}$ blank screen before the single center probe item was shown until a Same/Different response was made using a button box. Block 2B was identical to $2 \mathrm{~A}$ except that the mask screen was replaced with another $500 \mathrm{~ms}$ blank screen so that following the array of 5 colored squares participants were shown $1500 \mathrm{~ms}$ of blank screen prior to the center probe to be determined as Same/Different. Neither blocks 2A or 2B required the storage judgments to be made. Block $2 \mathrm{C}$ was identical to $2 \mathrm{~A}$ through the second 500ms blank screen, which was followed by a 3000ms screen with a "?" indicating the storage judgment should be made at that point. The Same/Different response immediately followed this $3000 \mathrm{~ms}$ storage judgment screen. Block $2 \mathrm{D}$ was an exact replication of Experiment 1 such that the storage judgment was to be made during a $4000 \mathrm{~ms}$ mask screen. (The only difference between 1 and 2D was that 1 required a storage judgment on only half of the trials whereas $2 \mathrm{D}$ required it on every trial.) In this way the timing of the visual array was consistent between the four blocks of trials in the following ways. (1) The Retention Interval (RI) between study and test for 2A and 2B was identical. (2) Block $2 \mathrm{C}$ had identical timing to $2 \mathrm{~A}$ and $2 \mathrm{~B}$ until the addition of the $3000 \mathrm{~ms}$ storage judgment screen. (3) The storage judgment of $2 \mathrm{C}$ was reduced from the $4000 \mathrm{~ms}$ for $2 \mathrm{D}$ in order that the RI for these blocks was identical. Recall that 2D (as in Experiment 1) did not have the second 500ms blank screen following the masks. Therefore the RI for $2 \mathrm{~A}$ and $2 \mathrm{~B}$ was $1500 \mathrm{~ms}$, whereas it was $4500 \mathrm{~ms}$ for $2 \mathrm{C}$ and $2 \mathrm{D}$.

\section{Auxiliary Tasks}


The Running Span and Letter Span tasks were identical to Experiment 1. The Ravens Progressive Matrices were now run on a computerized version rather than through a series of paper flashcards. Participants were left alone in the sound-attenuating booth to complete this portion after receiving instructions from the experimenter (though performance could still be monitored from outside of the booth).

\section{Results \& Discussion}

Experiment 2 was intended as a manipulation check to see if there were any additional changes that needed to be made to the study design in order for children to succeed at accomplishing the task. Four different versions of the task, described in the Experiment 2 Method section, were run on participants only from groups 1 and 4 of Experiment 1. A repeated measures ANOVA showed a significant effect of both Age Group, $F(1,50)=104.70, p<.0001, \eta_{p}^{2}=.68$, and Task Condition (i.e., different version of the task), $F(3,150)=17.18, p<.0001, \eta_{p}^{2}=.26$. However, there was no significant interaction between Group and Condition. Furthermore, a cursory glance at the Experiment 2 Means and Standard Errors in Figure 6 demonstrates that mean $k$ estimates did not differ within similar retention interval (RI) and storage judgment conditions. In other words, Conditions $2 \mathrm{~A}$ and $2 \mathrm{~B}$ each had a RI of $1500 \mathrm{~ms}$ and did not include storage judgments; whereas Conditions 2C and 2D each had a RI of 3000ms and did include storage judgments.

It should be noted that Experiment 1 showed no evidence that the storage judgments themselves interfere with change detection performance, when the judgments are interspersed throughout the trials. This may not remain the case when non-storage judgment trials are all blocked together. Overall, Experiment 2 provides evidence that the 
best study design for children completing this task is one in which storage judgments are blocked together, rather than interspersing them randomly with non-storage judgment trials. Overall, children continue to overestimate the number of items they have in working memory, however, it can be seen in Figure 7 that the blocking design improves the correlation between metamemory responses and mean $k$ estimates. The youngest Age Group shows the metamemory storage judgments and mean $k$ estimates have a Pearson's correlation of $r=.39$, whereas the adult group manages only a $r=.28$.

Additionally, Figure 8 shows that the RT data from Experiment 1 was replicated. Both age groups take about the same amount of time to provide their storage response, but the younger group still takes longer to provide their final change detection response. These results will be further examined in Experiment 3.

\section{Experiment 3}

Experiment 1 showed that children develop an increasingly accurate ability to estimate their own working memory contents with age. An increasingly shorter RT for making the same/different responses for the array probe was additionally shown. This increasingly shorter RT was not observed in making the storage judgments, which remained similar for groups of all ages. Experiment 2 replicated the results for the RT findings of Experiment 1. Experiment 3 will be a final study of the variable contributions of working memory capacity, processing speed, and quickness of forgetting. Here the participants will complete trials with varying array sizes and retention intervals (RI). If there is a pure capacity issue, participants will have trouble with larger array sizes irrespective of the RI. If the processing speed is the main issue, participants will have trouble with the shorter RI and the larger array sizes dependent upon the RI (i.e., a larger 
array with a shorter RI will be worse than a larger array with the longer RI). If the items are being forgotten too quickly, participants will have the most trouble on the longer RI as well as larger arrays. This experiment will also investigate the possibility that participant storage judgments are not an accurate reflection of what is in mind so much as a general tendency for 'middle ground' responding. The previous experiments show storage judgments of about three out of five items. The varying array sizes will be able to inform upon whether three items is an accurate assessment of personal working memory capacity, or if the participants simply tend toward a response in the middle between none of the array or all of the array.

The use of various array sizes will ensure that the participants' claim to have about three items in mind is based on actual ability, rather than a simple tendency to respond in the mid-range of the items. In other words, participants may claim three items in mind not because they actually know three items but because three is the middle response for an array of five. If this is the case, I would expect storage responses for arrays of 3, 6 and 9, to be 2, 3, and 4-5, respectively. I expect that the Group 1 children will have poorer performance (i.e., a lower estimated mean $k$ and higher storage judgments) for the longer RI showing evidence of quicker forgetting. Poorer performance of the shorter RI would alternatively be taken as evidence of slower processing. The remaining groups are expected to have comparably poorer performance on higher cognitive load trials but remain unaffected by the RI manipulation. It is possible Group 2 may show a transitory effect such that they are affected by RI to a lesser degree than Group 1 and affected by cognitive load to a lesser degree than the older age groups. The 
third possibility, a simple capacity difference based on age, would show improvements for all conditions as an effect of age group.

Overall, Experiment 3 is hypothesized to show that (1) storage judgments fail to show evidence of middle-ground responding, (2) all groups are able to provide accurate storage judgments in relation to mean $k$ except that (3) younger children have lower mean $k$ estimates on the longer RI condition despite higher storage judgments. In general, the adult groups are expected to perform relatively well in that their storage judgments should be closely similar to their mean $k$ scores. A larger array size is expected to result in a larger mean $k$ score for all age groups up to capacity. The middle age group is expected to approach the performance of the adult groups while the youngest group is expected to have particular difficulty with the longer RI. Quicker forgetting would be indicated by a lower mean $k$ score for the longer RI only for the youngest age group. In addition, this condition (4000ms RI for Group 1) is expected to have the highest storage judgments. These two results would indicate that the children likely did have more items in mind than will be evident through performance scores (indicated by the higher confidence shown by larger storage estimates), but that some of this information was lost from mind by the time the memory task was presented (resulting in the lower mean $k$ estimates).

Taken together, the three experiments in the current study will provide useful insight into an individual's awareness of their own cognitive processing. Experiment 1 showed that younger children have trouble estimating their own working memory capacity. However, the overestimations were still useful in predicting mean $k$ estimates despite this difficulty. Experiment 2 showed that the difficulty in making the personal 
capacity estimates can be alleviated if the task itself remains consistent within a block (i.e., young children make more accurate estimations if they know that they will be required to make the estimations on every trial rather than switching back and forth between trials that require estimates and trials that do not). Experiment 3 will shed light on the actual cognitive processing that leads to overestimates. In particular, it will provide an indication of why young children have lower working memory capacities than adults. Gathercole, Pickering, Ambridge, and Wearing (2004) suggest the structure of working memory is fully developed by age 6 despite the lower working memory capacity observed in children this young. These experiments attempt to ascertain whether the increasing capacity with development is the result of improvements in processing speed or retention of the information for a longer duration.

\section{Participants}

Experiment 3 used three age groups based on grade level. Group 1 included 20 children who were in $1^{\text {st }}-2^{\text {nd }}$ grade (mean age $=6.90$ years, 8 female). Group 2 included

23 children who were in $3^{\text {rd }}-7^{\text {th }}$ grade (mean age $=10.78,9$ female. Group 3 comprised 30 young adults (mean age $=23.50,24$ female) enrolled as undergraduate students at the University of Missouri. Undergraduate participants were given either $\$ 15$ or extra credit for a Psychology course. All children were paid $\$ 15$ dollars for their participation and were additionally given a book to take home.

\section{Procedure}

All tasks were completed in one of two sound-attenuating booths.

\section{Visual Array}


Experiment 1 provided evidence that young children were able to accomplish the storage judgment portion of the task that had not been used on participants so young in the past and that these introspective judgments appear to be useful in gaining knowledge additional to $k$ estimates. Experiment 2 showed that the youngest school-aged children could produce more accurate storage judgments if they were given foreknowledge that the storage judgment trials would all be blocked together rather than occur on a trial-bytrial basis. It was additionally shown that the mask screens, contrary to participant selfreports, did not interfere with memory or introspective ability. Based on $k$ estimates for trials with and without storage judgments, it is reasonable to assume RI causes memory difficulty in the youngest children, rather than the process of making the storage judgments (i.e., Experiment 2 showed $k$ estimates were lower for longer RI, but Experiment 1 showed no difference based on trials with or without storage judgments).

Experiment 3 presented all trials with the storage judgment portion of the task. There were six blocks of trials based on retention interval (RI) and array size. Blocks had a RI of $2000 \mathrm{~ms}$ or $4000 \mathrm{~ms}$ and array sizes of 3,6 , or 9 colored squares in order to investigate the effects of RI and cognitive load.

\section{Auxiliary Tasks}

The Running Span and Letter Span tasks were identical to Experiments 1 and 2. The Ravens Progressive Matrices were run using the same computerized version as Experiment 2. Participants were left alone in the sound-attenuating booth to complete this portion after receiving instructions from the experimenter (though performance could still be monitored from outside of the booth).

\section{Results \& Discussion}


Mean metamemory storage judgments and estimated $k$ scores are presented in Figures 9 and 10, respectively. The first question for Experiment 3 was a check on the participant's method for making their memory estimates. Specifically, there was a concern that participants may not be making earnest attempts for the estimates, rather they may simply have been responding in the middle of the range of possible judgments. Therefore, the accurate estimates of about 3-4 items may simply have been the result of 'middle ground' responding between 0-5 for the set size of 5 for both Experiments 1 and 2. Experiment 3 clearly shows that this was not the case. Nearly all participants estimated their working memory to include all 3 items for set size 3 . Set size 6 shows a similar, but slightly higher, estimate to the earlier set size 5 trials. Finally, set size 9 is only marginally higher than set size 6 . The expectation for middle ground responding was mean storage judgments of 2, 3, and 4-5 for the three set sizes. Across all age groups; set size 3 had a mean storage judgment of 2.96 items, set size 6 had a mean of 4.41, and set size 9 had a mean of 5.05 .

All groups overestimated their working memory in all three set sizes and for both retention intervals (RI). However, a multiple regression showed that the six conditions of storage judgments (by set size and RI) were a significant predictor of the individual condition's mean $k$ estimates despite the overestimations, $F(6,61)=3.51, p<.01$. It should be noted, however, that nearly all participants claimed all 3 items to be in mind for both set size three conditions. This may have weighted these results in favor of significance. The auxiliary measures of the Running Span, Ravens Progressive Matrices, and Letter Span tasks were run in order to test for the possibility that those participants who score higher on these standardized measures of working memory may have been able to make 
more accurate estimates of their own working memory contents. A multiple regression for storage judgments based on these tasks was not shown to be significant, $F(3,64)=1.73, n s$. This is not thought to be an indication of failure of these measures as the same regression for mean $k$ was significant, $F(3,65)=33.36, p<.0001$.

The final hypothesis of Experiment 3 was that the youngest age group would have lower mean $k$ estimates on the longer RI (see Figure 9 as a reference for the following paragraph). The mean $k$ estimates of this group did not differ as a function of RI on the smaller set size. However, the mean $k$ of Group 1 for RI $4000 \mathrm{~ms}$ was .39 items, whereas the mean $k$ for the $2000 \mathrm{~ms}$ RI was 1.14 items. A similar, non-hypothesized, result was shown in set size 6 of Group 2, in which mean $k$ for the $4000 \mathrm{~ms}$ RI was 1.47 items, whereas mean $k$ for the $2000 \mathrm{~ms}$ RI was 2.22 items. It was predicted that the older groups would perform better on the longer RI under the assumption that they could use the extra time for encoding the items while the youngest children would actually lose items they may have had in mind over the additional time. This result was shown in set size 9 of the young adults (Group 3), in which mean $k$ for the $4000 \mathrm{~ms}$ RI was 2.88 items but only 2.25 for the $2000 \mathrm{~ms}$ RI. 


\section{General Discussion}

A central question for this project was whether or not younger children overestimate their own working memory contents to a greater degree than older children and adults. Experiment 3 replicated both Experiments 1 and 2 in showing that all age groups appear to overestimate their own memory contents when compared to the commonly used $k$ estimates used by working memory researchers. However, the Experiment 3 results also demonstrate that $1^{\text {st }}$ and $2^{\text {nd }}$ grade children will continue to overestimate their memory ability well beyond the commonly theorized capacity limit of 4 items for working memory (e.g., Cowan, 2001), whereas older children appear to recognize their limitations for larger set sizes (e.g., 9 items) similar to young adults (see Figure 9). Experiment 2 showed that younger children could more reasonably estimate their working memory contents when storage judgments were blocked together (as opposed to the random requirement to make storage judgments on Experiment 1). Experiment 3 demonstrates that another important factor for young children's accuracy in making their estimates is a consistent set size. All trials in Experiment 2 used a set size of 5, whereas Experiment 3 varied set size from 3, 6, or 9 items. The younger children were unable to make accurate estimates despite the set sizes being presented in blocks rather than randomly varying on a trial-by-trial basis.

In the present study it was proposed that younger children have difficulty estimating the number of visual items in mind because of difficulty in retention of memoranda until a memory test can be administered. The three experiments from this project set out to test what the underlying mechanism is for why children are consistently shown to have a lower working memory capacity and reduced working memory abilities 
(e.g., inhibition, task switching, updating) compared to adults. The three possibilities proposed were that children's worse performance is the result of (1) a structural limit leading to lower capacity of working memory, (2) slower processing of working memory, or (3) quicker forgetting of working memory. The first possibility is unlikely for the current project as Gathercole, Pickering, Ambridge, and Wearing (2004) has shown evidence that the basic structure of working memory is complete by age 6 . All children in this project were at least 6 years old. The final two possibilities can be very simply examined merely by using memory tasks that allow varying amounts of time for encoding. Hypothesis 2 would be supported if younger children were shown to perform better with a longer retention interval (RI). Hypothesis 3 predicts the opposite; that younger children will perform better with a shorter RI.

Between all of the experiments in this project there are four RIs used. Experiment 1 always used a set size of 5 and a RI of $4500 \mathrm{~ms}$, as well as requiring the extra processing burden of storage judgments on a trial-by-trial basis. As mentioned in the Results \& Discussion section for Experiment 2, blocking the storage judgments led to more accurate judgments, indicating less processing may have been required during the RI for Experiment 2 compared to Experiment 1. Experiment 1 essentially required a degree of task-switching as individual trials had different requirements during the RI. The $1^{\text {st }}-2^{\text {nd }}$ grade children had an estimated $k$ of 1.14 items in this experiment, whereas the young adult $k$ was estimated to be 2.78 items. Experiment 2 always used a set size of 5, and either a RI of $4500 \mathrm{~ms}$ or $1500 \mathrm{~ms}$ based on whether or not the storage judgments had to be made during that block of trials or if a storage judgment did not have to be made for the that block, respectively. In this experiment, the $1^{\text {st }}-2^{\text {nd }}$ grade children had an estimated 
$k$ of .75 items for the $4500 \mathrm{~ms} \mathrm{RI}$, whereas the young adult $k$ was 2.97 . In other words, the young adults were able to achieve a higher $k$ score when (I conjecture) they had less processing to do in the same amount of time, whereas the opposite is true for the young children.

The use of different RI and cognitive load has previously been shown by Camos and Barrouillet (2011) to provide evidence for a shift in maintenance strategy between the ages of 6 and 7. This study showed that 6 year old children appear to utilize a passive maintenance strategy such that these children's performance was completely dependent on the duration of a task (i.e., the RI), whereas the 7 year old children are more likely to engage in some form of active maintenance such as refreshing the memoranda during the processing portion of the task. In the latter case, the children were determined to be able to divert attention away during a processing task such that their performance was no dependent on the cognitive load of a task rather than simply the RI. The present study replicated the negative effect of a longer RI on the youngest group of children.

It should be noted that the masks had no effect on the $k$ estimates, however, it could potentially be argued that a flaw in the current experiments is the differing presentation of the masks between experiments. It was possible that the masks could appear concurrently with the metamemory judgments (as in Experiments 1 and 3) or before the metamemory judgments were allowed to be made (as in Experiment 2), or with no masks at all. Previous research (Ricker \& Cowan, 2014; Ricker, 2015; Ricker \& Hardman, 2017) has shown that memory consolidation is not interrupted by the presentation of a mask in any of the above instances. In other words, having the masks on screen during or before the metamemory task should have no detrimental effect on the 
ability to consolidate the colors from the array. This effect was partially replicated in the present Experiment 2 in which the presence of a mask was shown to have no effect on capacity estimates, either through the subjective metamemory reports or the traditional $k$ values. The cited work above additionally showed that the duration of the masks' presence on screen had no effect on memory consolidation. Therefore the extended duration of the masks on screen (between $4000 \mathrm{~ms}$ and 500ms) should not negatively affect the participants' ability to consolidate the colors from the array. In addition, Saults and Cowan (2007) showed that, while a 600ms mask delay could reduce capacity estimates, this effect was unrelated to memory load such that it would interrupt processing. For the present study this means that the differing use of masks presentation onset and duration should have had no effect on the processing task of making the metamemory judgments and instead likely served only to erase any potential sensory memory from confounding the results.

At the least, because the young children actually did worse when they had less information to process, there is no evidence here to support the notion that the developmental improvement in working memory capacity is due to improved processing speed. Conversely, the young children actually achieved their highest estimated $k$ (1.57 items) on the shortest RI (1500ms) of any condition in this project. The young adults similarly achieved their highest $k$ (3.57) during this condition. Presumably, some of this effect is likely to be due to the fact that this condition was also the only one in the project to not include any storage judgments, and therefore required the least amount of processing during the RI. This was a main purpose of Experiment 3. 
The results of Experiment 3 in particular show some evidence that the young children are less able to maintain items for a longer RI (i.e., they suffer from quicker forgetting), rather than having difficulty processing the information fast enough for a shorter RI. In other words, Experiment 3 demonstrates that the $1^{\text {st }}-2^{\text {nd }}$ grade children performed better on the $1500 \mathrm{~ms}$ trials than the $4500 \mathrm{~ms}$ trial more because their memory was tested before they forgot the items than because they had less to process. Referring back to Figure 10, the only effect seen for RI was that the $1^{\text {st }}-2^{\text {nd }}$ grade children performed better when they a shorter RI for set size 9. As previously mentioned, the case could be made that the difference between the Experiment 1 and $2 k$ estimates for the $4500 \mathrm{~ms}$ was the result of varying amounts of processing required. However, both the $2000 \mathrm{~ms}$ and $4000 \mathrm{~ms}$ conditions of Experiment 3 required the same amount of processing during the RI. Set size 9 resulted in a $k$ of 1.14 items in the $2000 \mathrm{~ms}$ RI and only .39 items in the $4000 \mathrm{~ms}$ RI for $1^{\text {st }}-2^{\text {nd }}$ grade children. The opposite effect was observed in young adults, who had an estimated $k$ of 2.25 items in the $2000 \mathrm{~ms}$ RI and improved to 2.88 items in the $4000 \mathrm{~ms}$ RI.

The results above do not, and cannot, account for the structural hypothesis for increasing capacity in working memory from children to adults. The second hypothesis claiming the increased capacity is due to increasing the ability to more quickly process information appears to have only minimal support. There are two minor points in favor of this hypothesis from the adult population. First, the adults improved their performance between Experiments 1 and 2 when the only difference was the blocking of the storage judgment portion of the task. It could be conjectured that the adults, but not the children, benefited from not needing to worry about whether or not each individual trial required a 
storage judgment or not. Second, the longer RI of Experiment 3 could be said to have allowed additional encoding time for the adults. This may be why they improved their performance on the longer RI whereas the children became much worse.

The final hypothesis appears to be the best supported by the data in this project. The reason for the developmental increase in working memory capacity is that, as children age, they become better able to encode and retain visual information for a longer period of time. The adults tended to perform best when they had more time for encoding information. Conversely, the youngest children tended to perform better when they were tested on the visual items more quickly. The above discussion comprises the conclusions drawn from the mean $k$ results in relation the mechanism driving most of the improvement in working memory capacity from childhood to adulthood. However, the question of the utility of the introspective, subjective storage judgments remains.

Unfortunately, the results of the three experiments all seem to present conflicting pictures of the use of storage judgments in developmental research. The most successful attempt was Experiment 2. This experiment was also the simplest one of the project. The task itself seems to be one that children as young as 6-7 are capable of understanding only when it is presented without any major changes. Changing set size, RI, or task demands on a trial-by-trial basis all appear to have the effect of making the measure itself incomprehensible. There are, however, a few important points that can be gleaned from the results at large for the potential future use of storage judgments in working memory research. First, the amount of time given to make the judgments does not alter the estimates. Children overestimated to a similar degree, whether they had $2 \mathrm{~s}$ to respond, $4 \mathrm{~s}$ to respond, or had to wait $2 \mathrm{~s}$ before responding in a delayed $2 \mathrm{~s}$ interval. Second, young 
children will increase their estimates as long as the set size is increasing, whereas older children display more awareness of their own limitations. For example, the storage judgment results for $4^{\text {th }}-7^{\text {th }}$ grade children are nearly identical to the young adults for all set sizes of Experiment 3. Finally, as long as the measure is kept consistent and simple (as in Experiment 2), individual awareness about your own working memory contents can be marginally can be useful in determining ability on other working memory measures such as the Running Span, Ravens, and Letter Span tasks used in this project.

Some, or perhaps many, of the results in the present study can be interpreted through the idea of object files, or the process of setting up and utilizing temporary episodic representations of real world objects (Kahneman, Treisman, \& Gibbs 1992). A potentially relevant aspect of object files is that they are theorized to contain multiple properties built up into a single mental representation (See also the Appendix on Binding in the current paper). Kahneman, Treisman, and Gibbs posit that attention to any one property of an object will increase attention to other properties of an object, even if they are irrelevant. However, a recent perspective by Cowan (2016) claims that object files may not necessarily be filled with all of the properties of the real world object. Therefore, participants may be overestimating their capacity because they are aware of the locations of more than the objective $k$ estimate of the color-items, which then leads to partial attention given to the colors presented at those locations. The participant may then misinterpret their greater awareness of specific locations for the number of colors they memorized from the array. Previous work by Noles, Scholl, and Mitroff (2005) has shown that object-specific previewing benefits (OSPBs), or speeded judgments about properties of objects when those properties are previewed, may last for at least 8 seconds. 
In the current study, the masks are shown in the same locations as the array squares upon which the storage judgments are to be made for color. The masks themselves therefore may be directing attention to location properties leading to the previously mentioned misinterpretation of location awareness for color awareness. Infant research (Zosh \& Feigenson, 2012) suggests a developmental component to the loss of representational precision. This could explain the greater overestimation by the younger age groups. Another implication of the OSPB described by Noles, Scholl, and Mitroff (2005) and its potential relation to attention to one property increasing attention to another property (Kahneman, Treisman, \& Gibbs, 1992) despite the possibility that not all real world properties fill the object files (Cowan, 2016) is the idea of the illusion of knowing. The previous sentence sums up a similar effect that occurs on a larger scale. The illusion of knowing is an event that occurs when an individual's assumed knowledge of something such as a procedure or mechanical device hinders their ability to recognize their relative lack of knowledge following an explanation of an expert. While most adults appear to be show self-awareness following the explanation, children have more trouble (Mills \& Keil, 2004). This effect has been tested on elementary school children at least since Markman (1977) in which children were tested on their ability to memorize sentences. The results of this study showed that children as old as 12 were able to answer probed questions about the sentences they had read. Markman demonstrated that children were able to complete a task in which they were required to encode and store information, draw inferences, and retrieve and compare different propositions from previously read sentences. Despite this, however, the children, unlike the adults, were less able to recognize inconsistencies that arose. In effect, even though the children were 
able to carry out all of the necessary cognitive components of the task they continued to fail to notice additional, false information. The children in the current study were apparently capable of completing each aspect of the visual array and metamemory task, but may have similarly been unable to recognize when additional information was present or misinterpreted from adjacent properties of the estimated items (e.g., location information).

A potential use for the metamemory storage judgment methodology used in this project was identifying individual needs or skills in a practical, school setting. Adhering to the three points above would be necessary in making any such practical application viable. This project was not an easy one for children to complete. As stated in the intro, children with a lower working memory have a greater tendency to mind-wander (Kane \& McVay, 2012), whereas children with e higher working memory will mind-wander more often when a task is particularly easy (Levinson, Smallwood, \& Davidson, 2012). The current task has the ability to show which school children have lower working memory spans and would therefore be more likely to mind-wander. More importantly, for those children with a higher working memory span, the storage judgment portion of the task can be used to indicate which children are better or worse at monitoring their own working memory and may therefore would be better served in school with a more difficult curriculum.

One of the important aspects of the metamemory storage judgments is that the individual completing the task is required to predict their performance on a task. Most metamemory research, (e.g., confidence ratings) is retrospective of performance. Future work should look to combine these two methods. Many participants in the current project would 
greatly overestimate the earlier trials and then slowly bring their estimates down to a more reasonable level upon realizing they were not doing as well as they originally thought they might (i.e., after getting negative feedback more often than they were expecting). A potential future study could proceed as follows: (1) memoranda, (2) storage judgment, (3) memory test, (4) confidence rating, and (5) feedback. The extra confidence interval step could further improve the practical application of determining individual skill at monitoring performance on a cognitive task. An additional use for the inclusion of confidence rating would be to ask participants to rate the confidence they have in the accuracy of each metamemory judgment. Confidence ratings are typically used in order to gauge a participant's perceived confidence in their accuracy in correctly responding to a trial (e.g., as above, confidence that the "same/different" response to the probe was accurate). By instead obtaining a confidence in individual metamemory judgments it may be possible to discern whether or not participants are actually aware when they are overestimating. Future studies in which $k$ estimates are differentially obtained on high confidence versus low confidence trials may show that (even though participants tend to overestimate their own capacity) they have lower confidence in incorrect, high metamemory estimate trials, as opposed to correct, high metamemory trials. This could provide a level of insight into our own cognitive abilities surpassing previous confidence rating research as well as the current study. Currently, it remains to be seen if there is a relationship between confidence ratings and correcting overestimates of storage judgments. A final future avenue of research pertains to the issues discussed about attention to location properties rather than color properties. This potential issue could be investigated by marking the locations of the array items during the storage judgment 
portion of the task. Rather (or in addition) to incidentally directing attention to the locations of the array items, the locations could be systematically marked for two reasons. One reason would be two potentially avoid any participant confusion about what they are meant to be estimating. If the locations are clearly marked, they would presumably be less likely to mistake location rather than color as the key property they are meant to be estimating. Second, blocks of trials in which locations are marked or remain unmarked could provide insight into whether OSPBs are indeed influencing the overestimations. 


\section{APPENDIX A}

Binding affords the possibility to examine how individuals maintain multiple features of a single item as well as how well recognition of memoranda compares to a previously unobserved stimulus (e.g., a probe color that was nowhere in an array as opposed to a probe color that was observed but has mismatched another feature such as location).

Luck and Vogel (1997) asserted that the number of features present in a single stimulus item does not have an effect on working memory capacity as a whole. Zhang and Luck (2008) show further support of the claim of a discrete slot model of visual working memory. Each of these influential studies asserted that there are a discrete number of slots in working memory that remain unaffected by the complexity of the stimulus items. In other words, the number of items within the capacity of visual working memory remains constant (e.g., 4), regardless of whether you are trying to maintain fours simple colors or four colored shapes. Binding multiple features does not take up additional available discrete slots. Luck and Vogel's results have generated some level of controversy and had previously failed to replicate Wheeler and Treisman, (2002) and Delvenne \& Bruyer, (2004). However, recent work goes further to dispute the claim of complexity-irrelevant item capacity based on significant effects to the contrary, in addition to simply failing to replicate. Hardman and Cowan (2015) found that working memory capacity may well be dependent on item complexity when there are multiple features (e.g., line color and orientation) that require binding in working memory.

Hardman and Cowan (2015) found that a Bayesian model that incorporates feature-binding complexity better predicted data then similar models that do not take 
complexity into account. Additional evidence for such an effect has had been postulated by others (Cowan, Blume, \& Saults, 2013; Oberauer \& Eichenbereger, 2013, Zosh \& Feigenson, 2015). Cowan, Blume, and Saults surmise that item complexity plays a role in working memory capacity in that the overall capacity can remain consistent for simple objects and those with multiple complex features due in part because of incomplete encoding of features. Capacity may remain constant at about 4 items total given that an individual may know both features of an item while perhaps only knowing a single feature of two additional items. The partial information can then be used to assist in guessing by striking off any potential memoranda that don’t fit the partial knowledge. For example, an individual may be able to correctly deduce an array did not contain a green circle because of knowledge of a different item that was a green square. Full feature knowledge of one object can provide crucial information for others even though the individual is not able to directly report the absence of a green circle. Cowan, Saults, and Blume (2014) provide additional support by postulating a central and a peripheral component of working memory for both verbal and visual information. These reports suggest (cf. Hardman \& Cowan, 2015) that a logical step forward for working memory capacity research would be best to forge ahead with attempts to improve current knowledge about feature-binding complexity contributions rather than a strict attempt to make a clear decision on which of the many factors is most important to determining capacity. 


\section{APPENDIX B}

(1a)

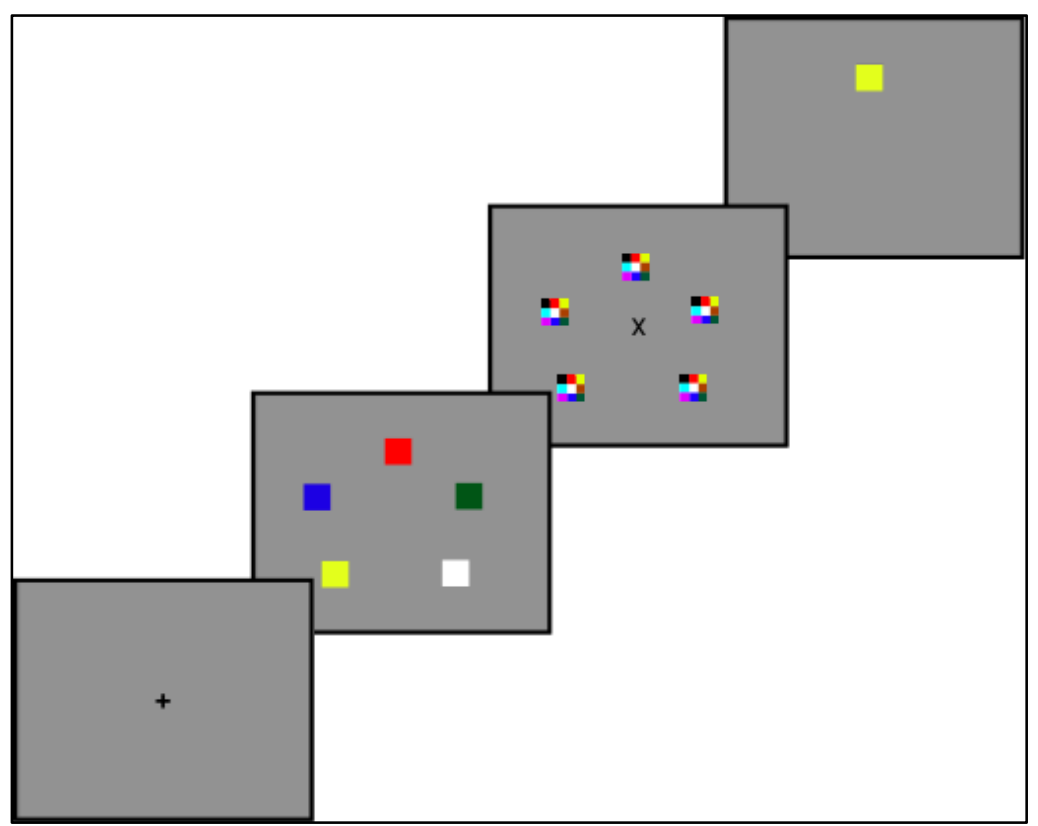

(1b)

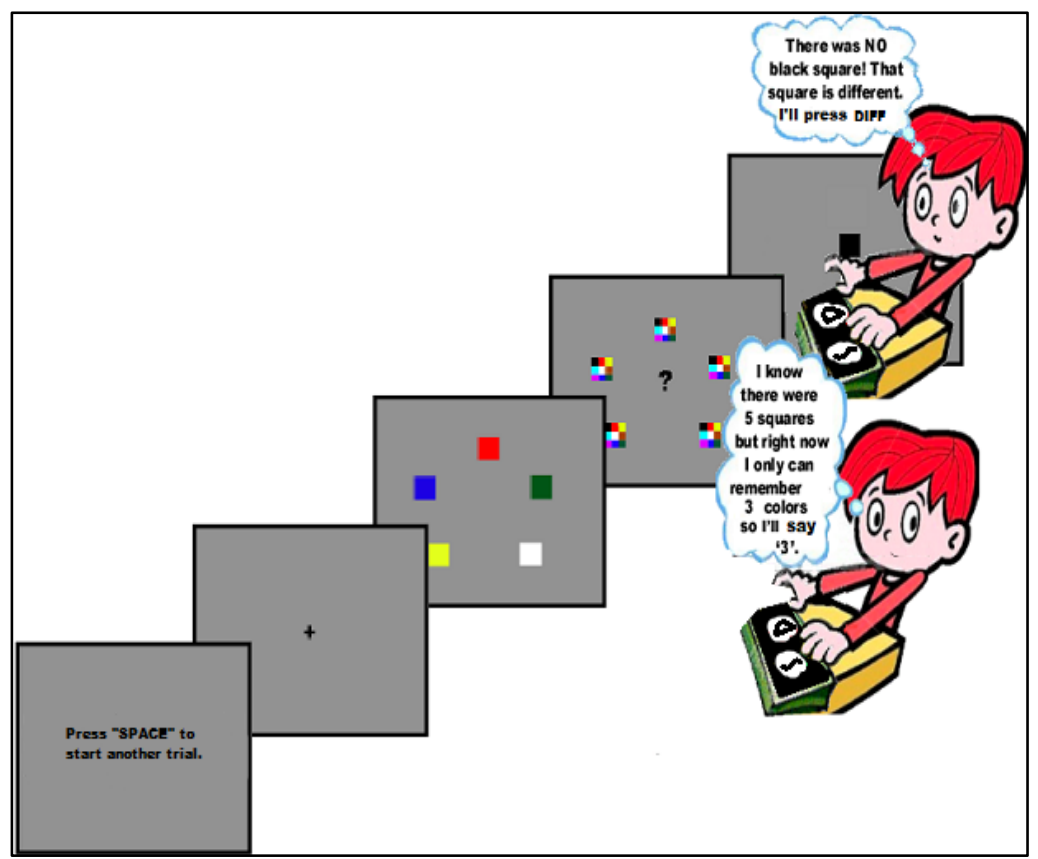

Figure 1. Design for Experiment 1 without (1a) and with (1b) metamemory storage judgment. 


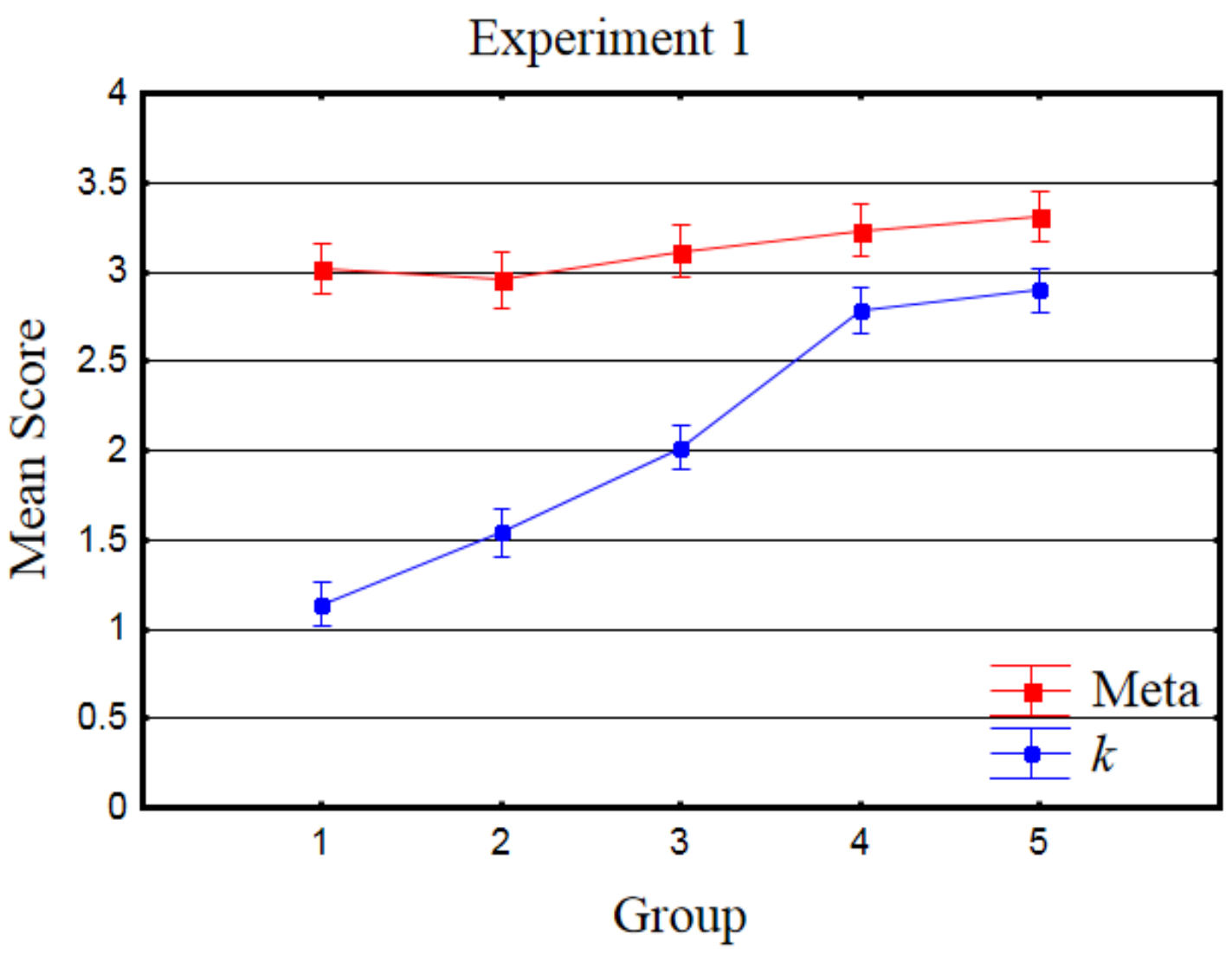

Figure 2. Experiment 1 mean estimates of capacity (k) and storage judgments (Meta). 


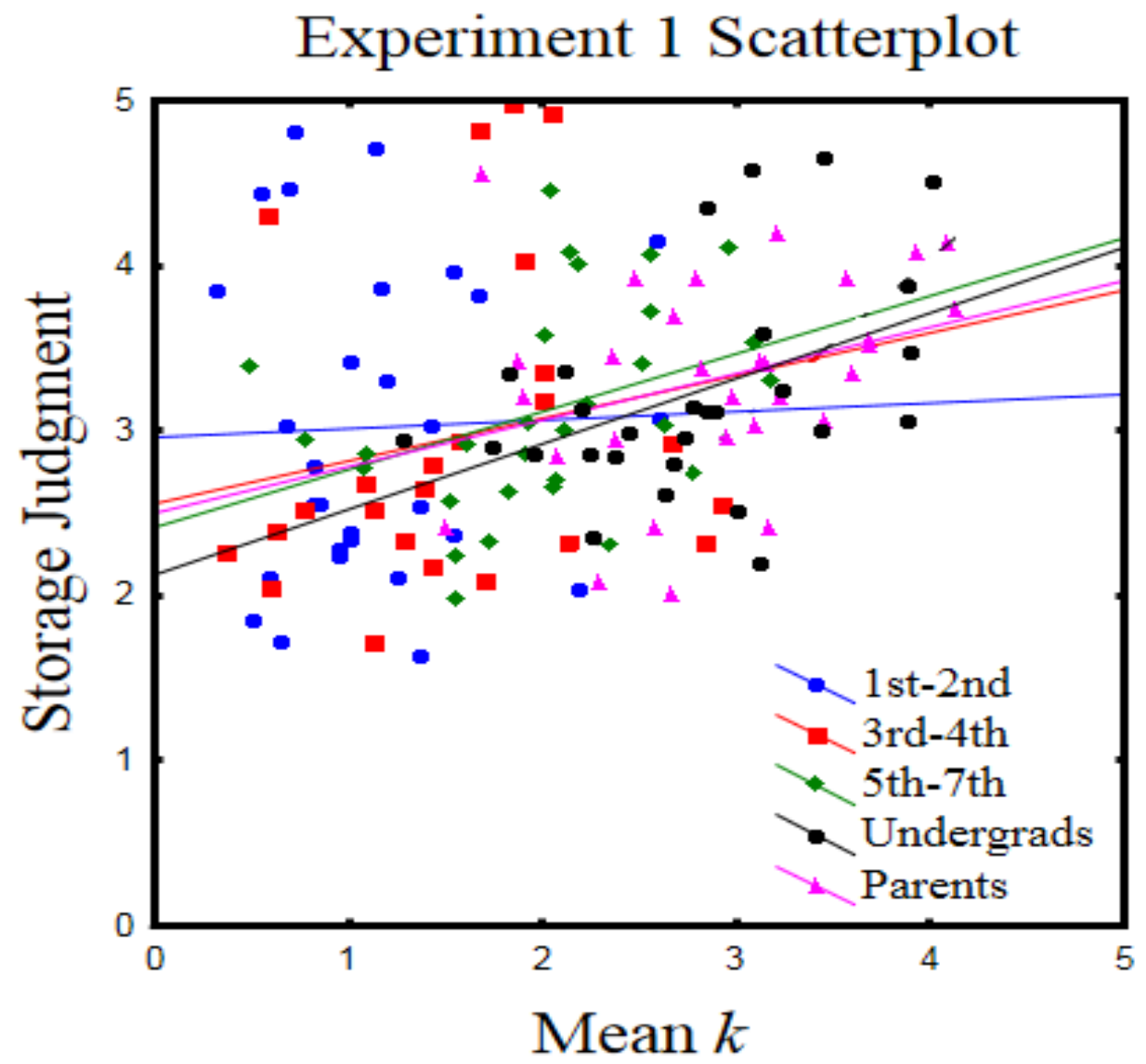

Figure 3. Experiment 1 scatterplot of participants' mean capacity (k) and mean storage judgment. 


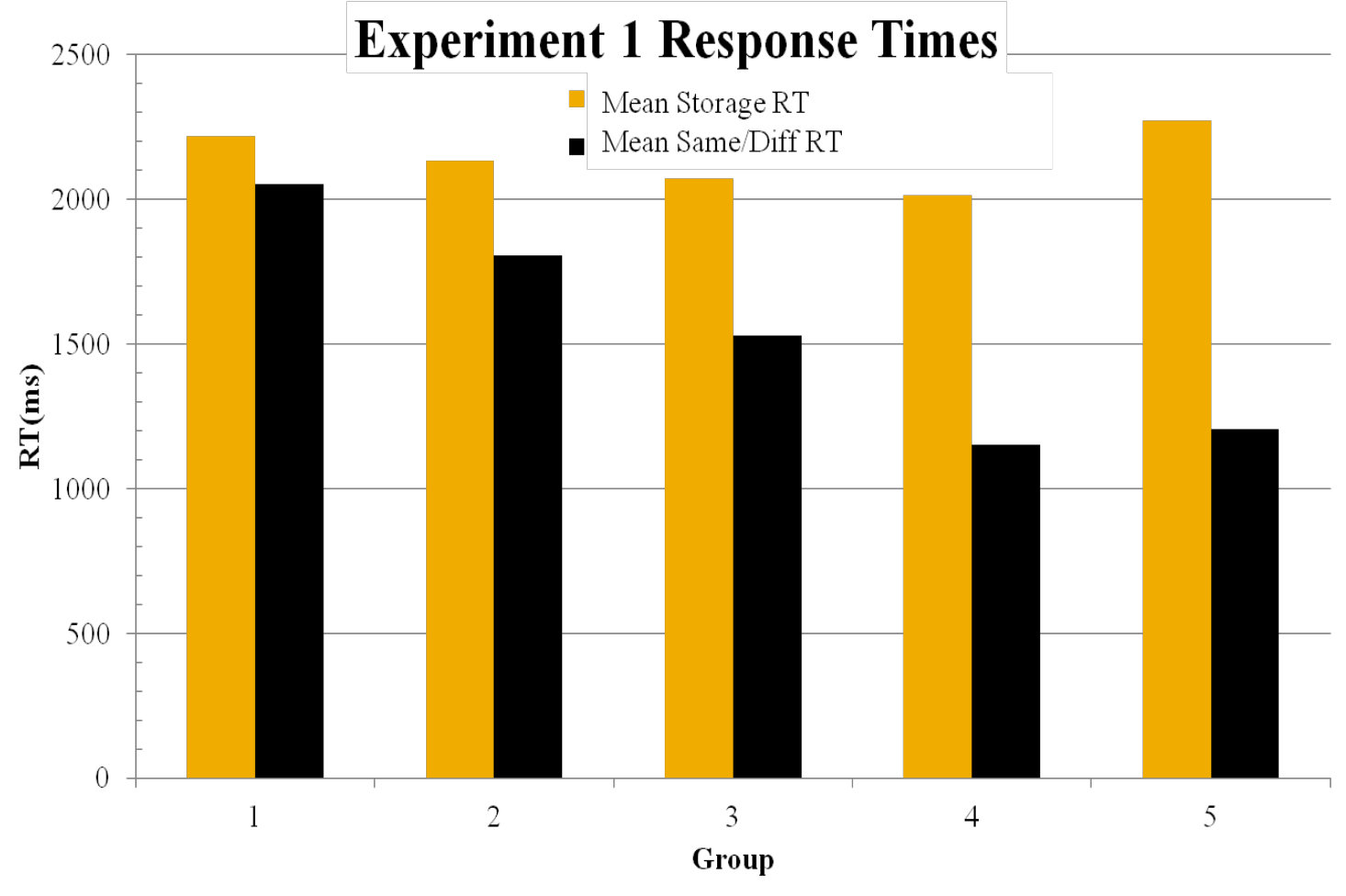

Figure 4. Experiment 1 response times (RT) for providing a verbal storage judgment and for responding to the probe as Same or Different. Note. Storage judgment RT includes the time it took an experimenter to provide a key press response following a participant's verbal indication of storage. 

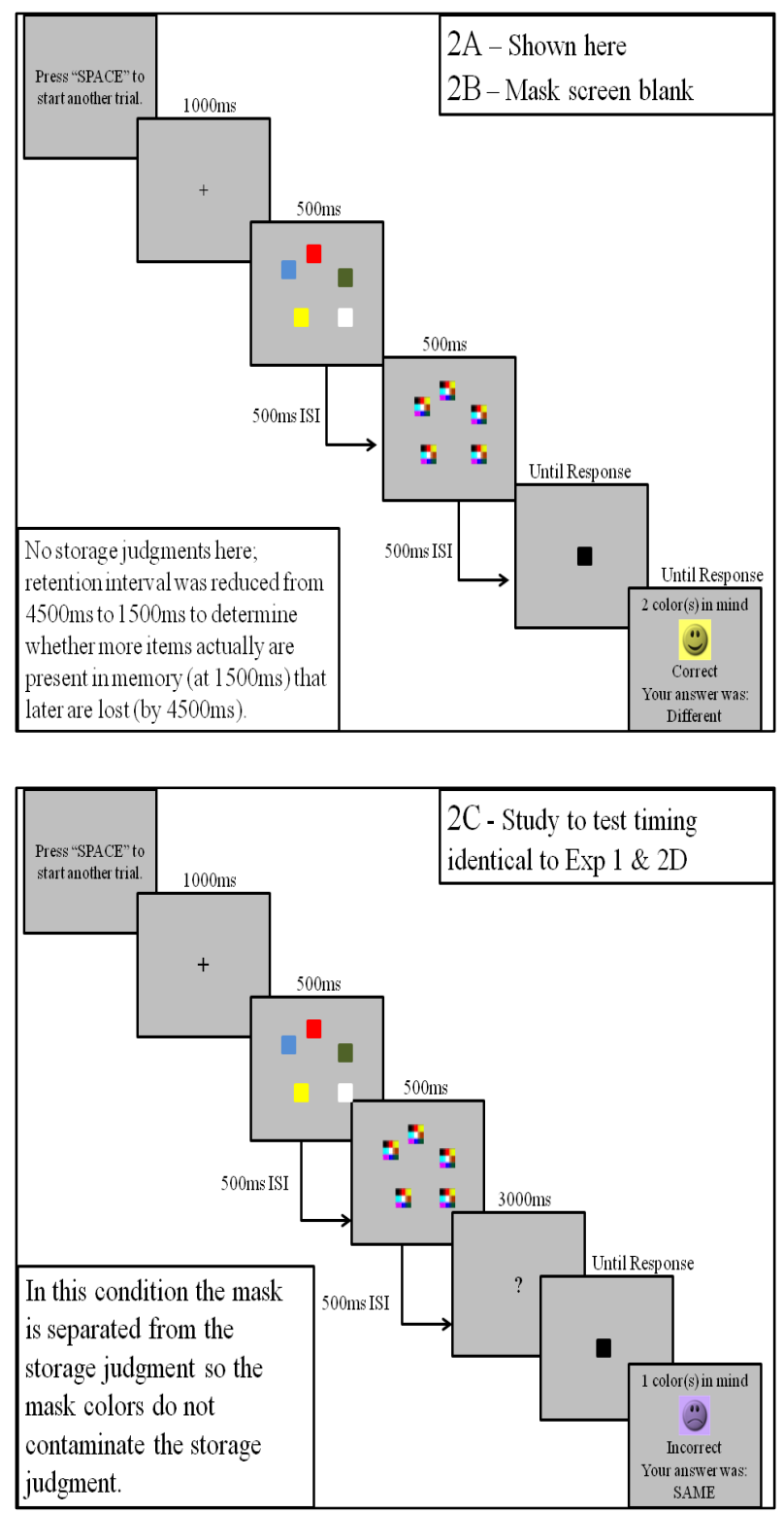

Figure 5. Design for Experiment 2 conditions without (left panel) and with (right panel) storage judgment. 


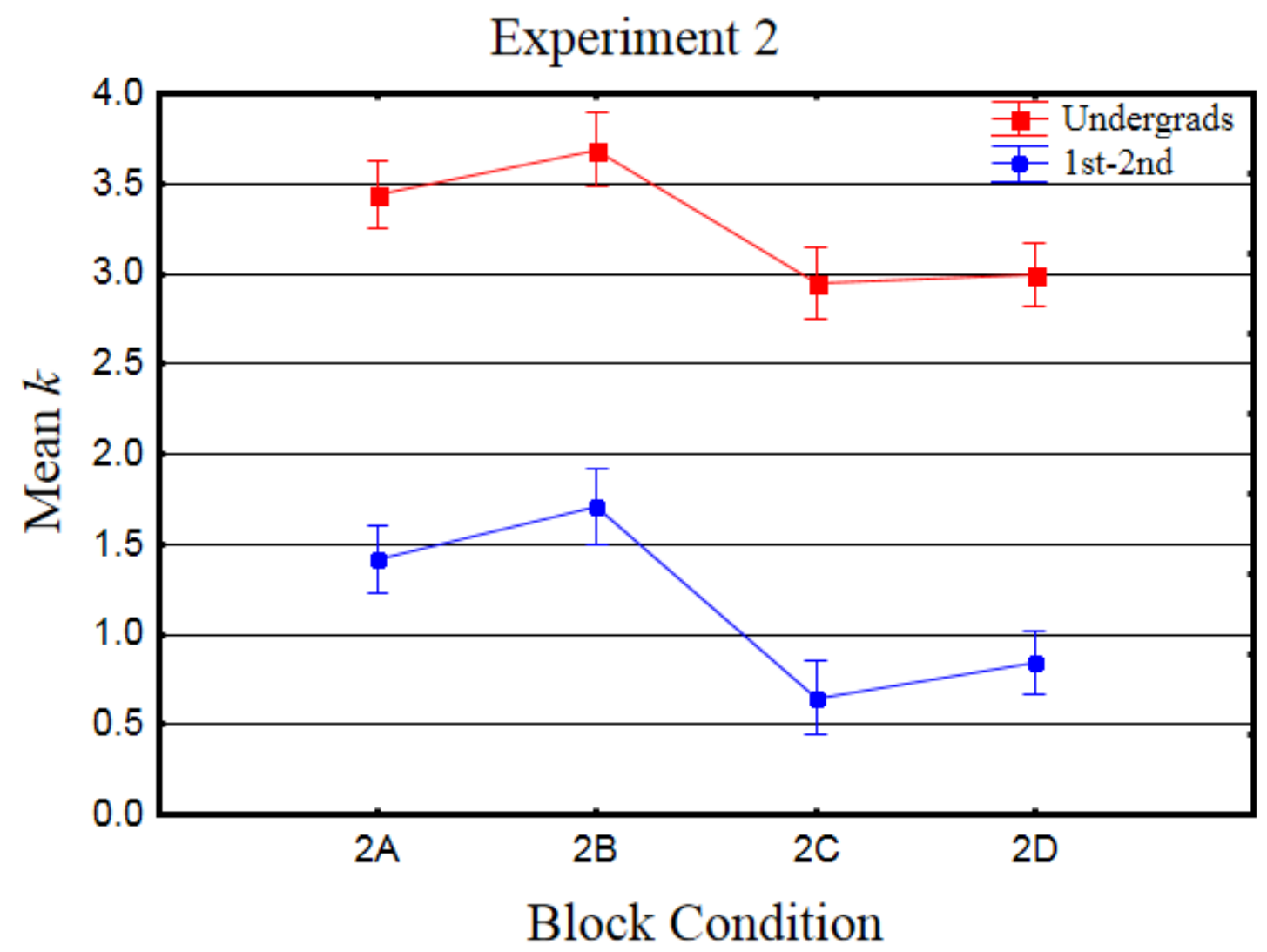

Figure 6. Experiment 2 mean capacity (k) estimates by group and condition. 


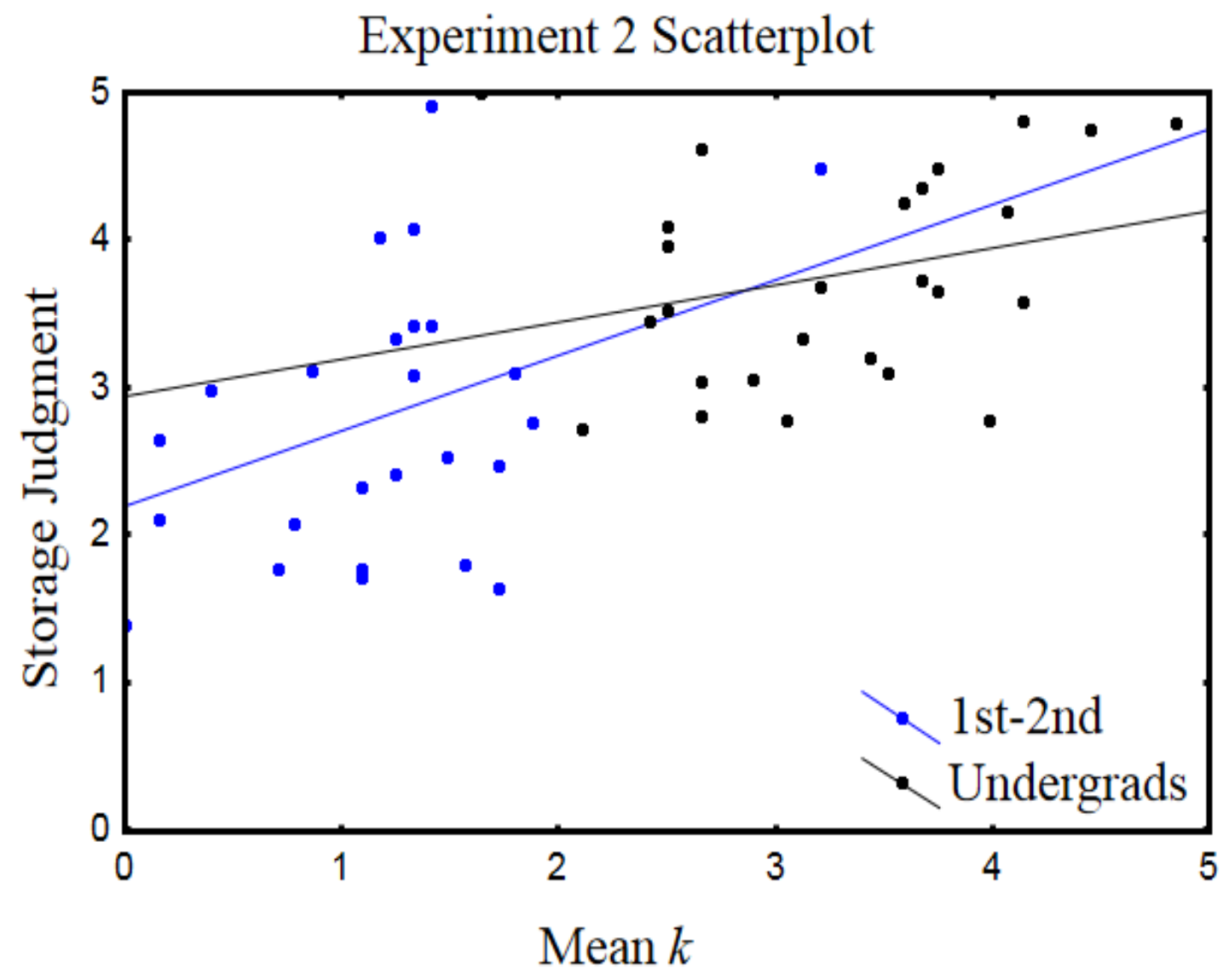

Figure 7. Experiment 2 scatterplot of participants' mean capacity (k) and mean storage judgment. 


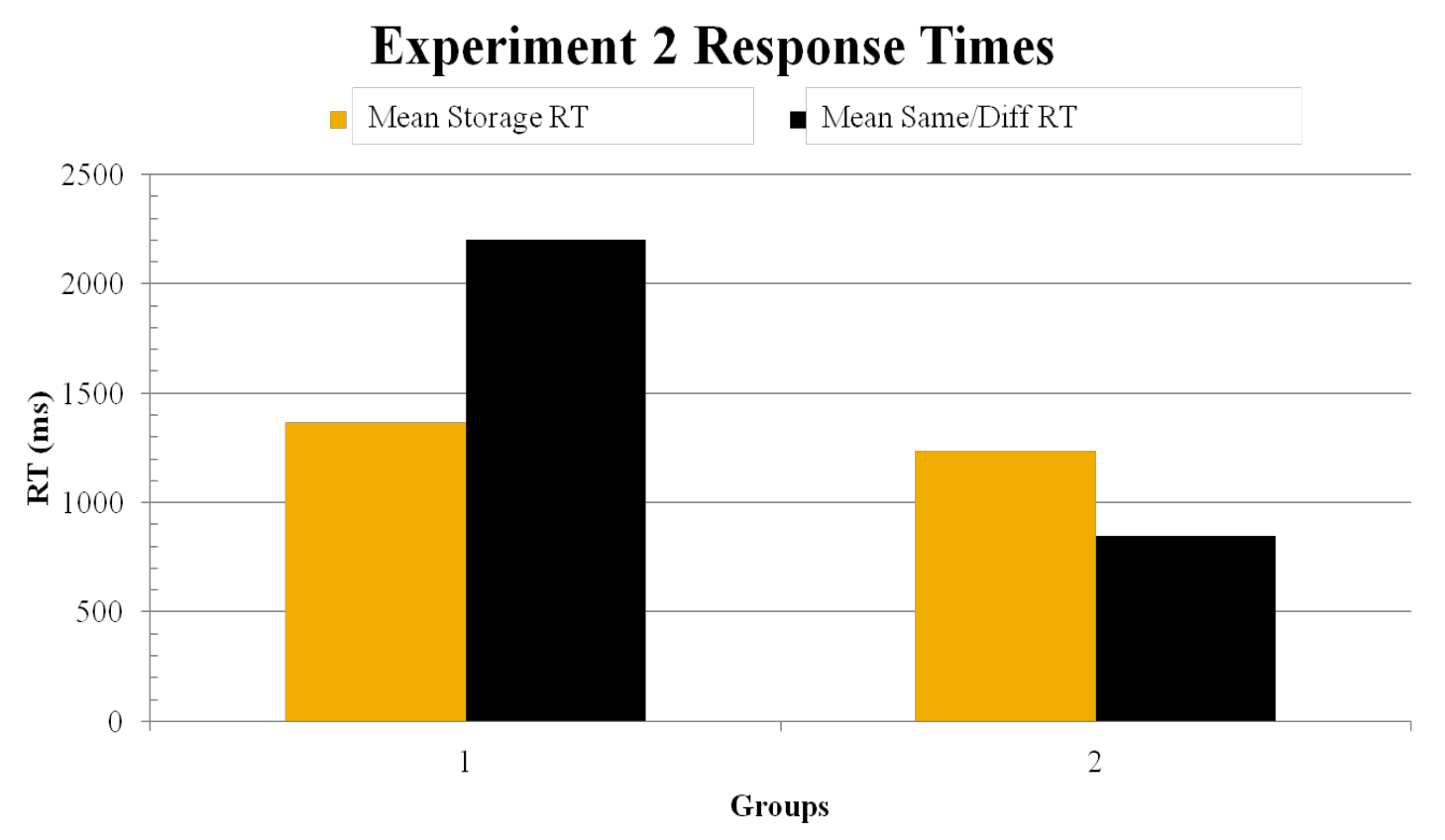

Figure 8. Experiment 2 response times (RT) for providing a verbal storage judgment and for responding to the probe as Same or Different. Note. Storage judgment RT includes the time it took an experimenter to provide a key press response following a participant's verbal indication of storage. 


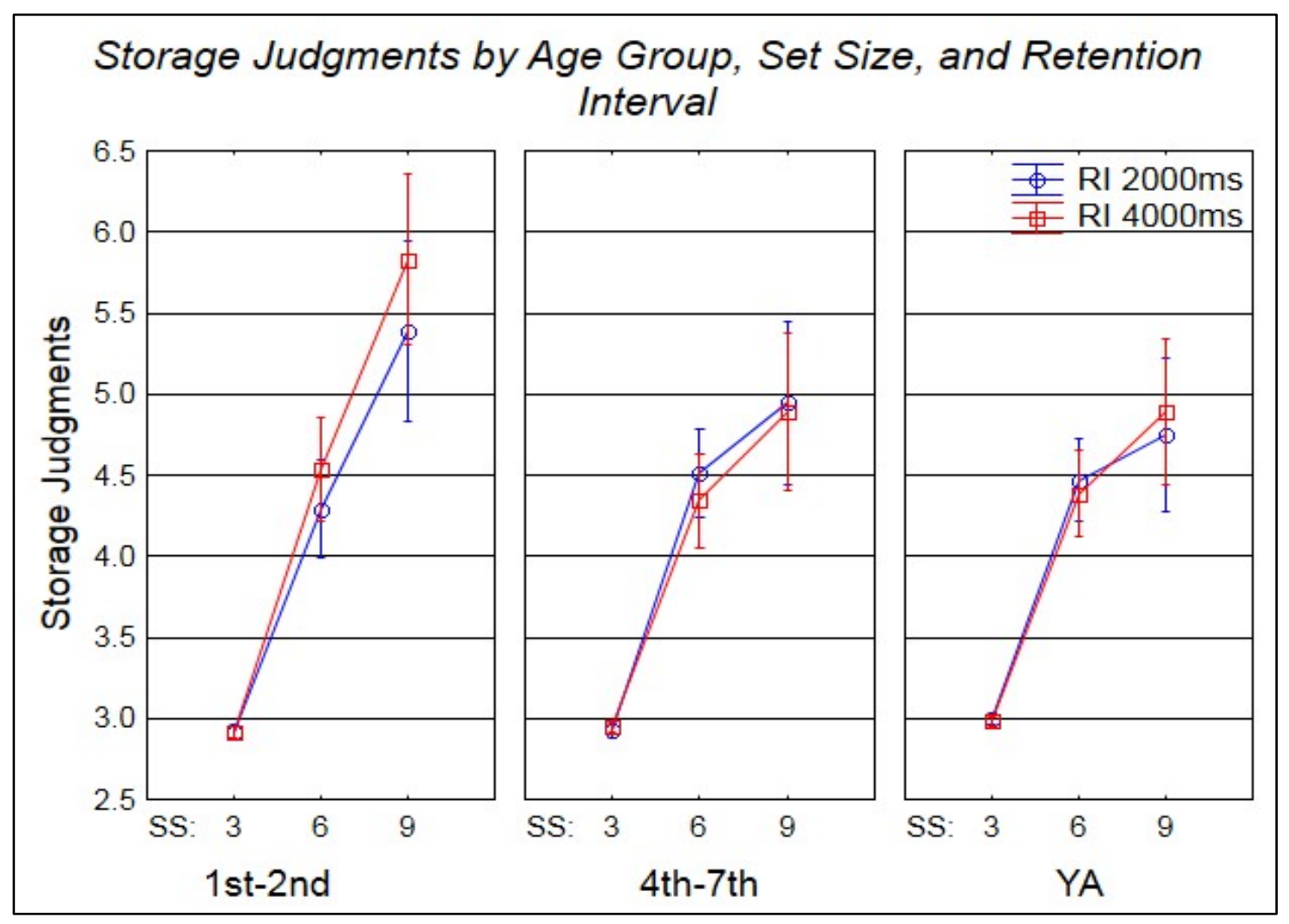

Figure 9. Experiment 3 mean results for metamemory Storage Judgments (with SEM) by Age Group, Set Size (SS), and Retention Interval (RI). 


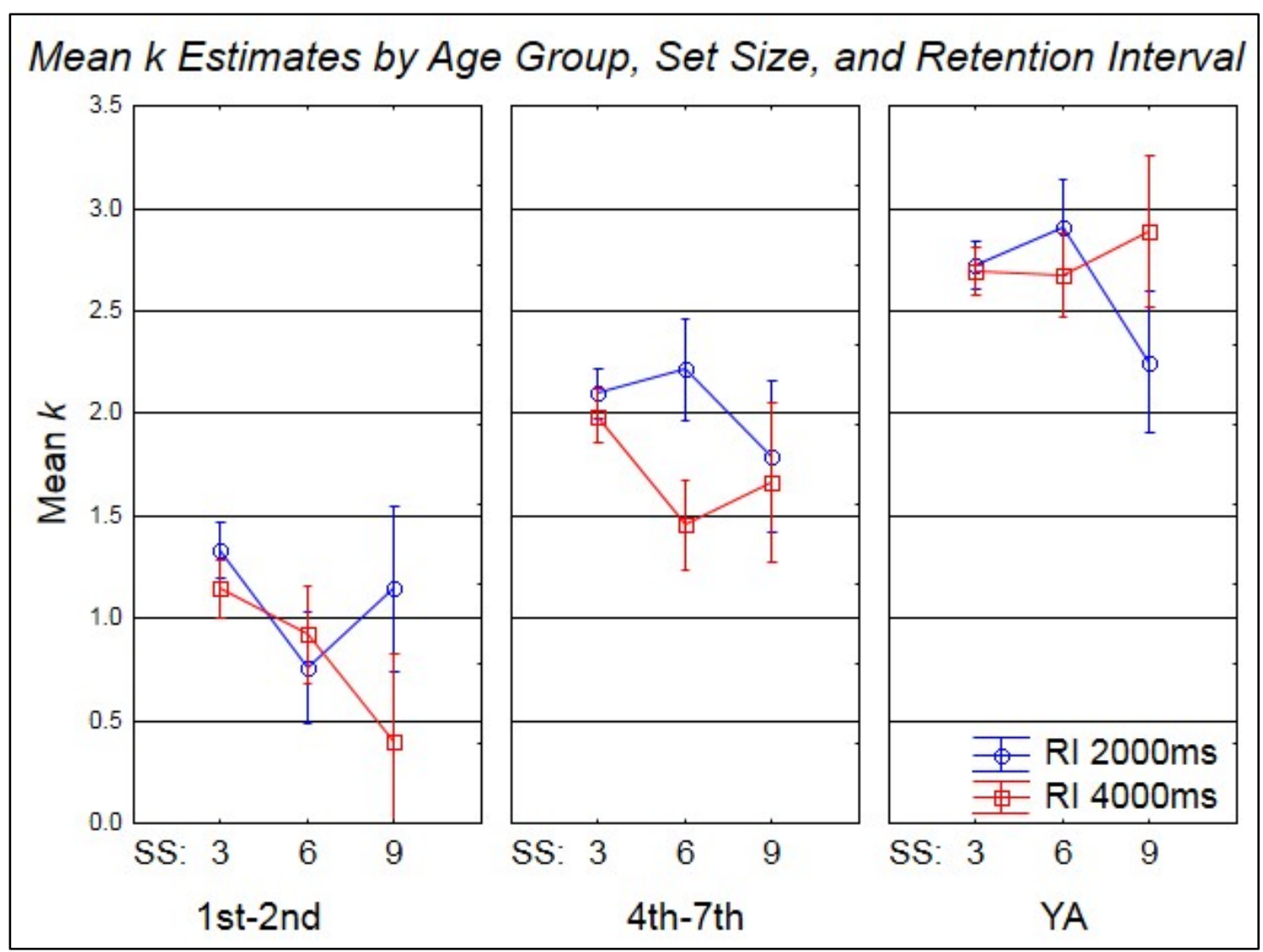

Figure 10. Experiment 3 mean results for Mean $k$ estimates (with SEM) by Age Group, Set Size (SS), and Retention Interval (RI). 


\section{APPENDIX C}

Table 1.

Experiment 1 measurement means (with SEM) by age Group. Mean k was estimated using the formula $k=N(h-f a)$ where $N$ is the array size, $h$ is proportion of hits (change detected), and fa is proportion of false alarms (change falsely detected). Note. YA and OA stand in for Young Adults and Older Adults.

$\begin{array}{cccccc} & \text { 1st-2nd } & \text { 3rd-4th } & \text { 5th-7th } & \text { YA } & \text { OA } \\ \text { meank } & 1.14(.11) & 1.54(.14) & 2.02(.12) & 2.78(.13) & 2.90(.13) \\ \text { meang } & 0.36(.02) & 0.31(.02) & 0.31(.02) & 0.24(.02) & 0.28(.02) \\ \text { Meta } & 3.02(.18) & 2.96(.20) & 3.12(.12) & 3.23(.12) & 3.32(.11) \\ \text { Ravens } & 26.83(1.79) & 33.62(1.93) & 39.97(1.30) & \mathrm{N} / \mathrm{A} & 49.13(1.17) \\ \text { RunningSpan } & 1.90(.14) & 2.37(.15) & 2.68(.18) & 2.86(.12) & 3.13(.13) \\ \text { LetterSpan } & 0.62(.11) & 1.18(.13) & 1.92(.18) & 2.42(.17) & 2.61(.17)\end{array}$




\section{REFERENCES}

Bae, G.Y., \& Flombaum, J.I. (2013). Two items remembered as precisely as one: How integral features can improve visual working memory. Psychological Science, 24(10), 2038-2047.

Bays, P.M., \& Husain, M. (2008). Dynamic shifts of limited working memory resources in human vision. Science, 321(5890), 851-854.

Borella, E., \& De Ribaupierre, A. (2014). The role of working memory, inhibition, and processing speed in text comprehension in children. Learning and Individual Differences, 34, 86-92.

Camos, V., \& Barrouillet, P. (2011). Developmental change in working memory strategies: From passive maintenance to active refreshing. Developmental Psychology, 47(3), 898-904.

Courage, M., \& Cowan, N. (Eds.). (2008). The development of memory in infancy and childhood. Ney York, NY, US: Psychology Press.

Cowan, N. (2001). Metatheory of storage capacity limits. Behavioral and Brain Sciences, 24(1), 154-176.

Cowan, N. (2014). Working memory underpins cognitive development, learning, and education. Educational Psychology Review, 26(2), 197-223.

Cowan, N. (2016). Working memory maturation: Can we get at the essence of cognitive growth? Perspectives on Psychological Science, 11(2), 239-264.

Cowan, N., \& Alloway, T. (2009). Development of working memory in childhood. In M. L. Courage \& N. Cowan (Eds.), Studies in developmental psychology. The development of memory in infancy and childhood (pp. 303-342). New York, NY, US: Psychology Press.

Cowan, N., \& Morse, P.A. (1986). The use of auditory and phonetic memory in vowel discrimination. The Journal of the Acoustical Society of America, 79(2), 500-507.

Cowan, N., Blume, C.L., \& Saults, J.S. (2013). Attention to attributes and objects in working memory. Journal of Experimental Psychology: Learning, Memory, and Cognition, 39(3), 731-747.

Cowan, N., Elliott, E.M., Saults, J.S., Morey, C.C., Mattox, S., Hismjatullina, A., \& Conway, A.R. (2005). On the capacity of attention: Its estimation and its role in working memory and cognitive aptitudes. Cognitive Psychology, 51(1), 42-100. 
Cowan, N., Hardman, K., Saults, J.S., Blume, C.L., Clark, K.M., \& Sunday, M.A. (2016). Detection of the number of changes in a display in working memory. Journal of Experimental Psychology: Learning, Memory, and Cognition, 42(2), 169-185.

Cowan, N., Saults, J.S., \& Blume, C.L. (2014). Central and peripheral components of working memory storage. Journal of Experimental Psychology: General, 143(5), $1806-1836$.

Daneman, M., \& Carpenter, P.A. (1980). Individual differences in working memory and reading. Journal of Verbal Learning and Verbal Behavior, 19(4), 450-466.

Delvenne, J.F., \& Bruyer, R. (2004). Does visual short-term memory store bound features? Visual Cognition, 11(1), 1-27.

Fougnie, D., \& Marois, R. (2011). What limits working memory capacity? Evidence for modality-specific sources to the simultaneous storage of visual and auditory arrays. Journal of Experimental Psychology: Learning, Memory, and Cognition, 37(6), 1329-1341.

Gathercole, S.E., Pickering, S.J., Ambridge, B., \& Wearing, H. (2004). The structure of working memory from 4 to 15 years of age. Developmental Psychology, 40(2), 177-190.

Gulbinaite, R., Johnson, A., de Jong, R., Morey, C.C., \& van Rijn, H. (2014). Dissociable mechanisms underlying individual differences in visual working memory capacity. Neuroimage, 99, 197-206.

Hardman, K.O., \& Cowan, N. (2015). Remembering complex objects in visual working memory: Do capacity limits restrict objects or features? Journal of Experimental Psychology: Learning, Memory, and Cognition, 41(2), 325-327.

Hellström, Å. (1985). The time-order error and its relatives: Mirrors of cognitive processes in comparing. Psychological Bulletin, 97(1), 35-61.

Hollingworth, H.L. (1910). The central tendency of judgment. The Journal of Philosophy, Psychology and Scientific Methods, 7(17), 461-469.

Holmes, J., \& Gathercole, S.E. (2014). Taking working memory training from the laboratory into schools. Educational Psychology, 34(4), 440-450.

Holroyd, C.B., \& Coles, M.G. (2002). The neural basis of human error processing: reinforcement learning, dopamine, and the error-related negativity. Psychological Review, 109(4), 679-709.

Kahneman, D., Treisman, A., \& Gibbs, B.J. (1992). The reviewing of object files: Object-specific integration of information. Cognitive Psychology, 24(2), 175-219. 
Kane, M.J., \& McVay, J.C. (2012). What mind wandering reveals about executivecontrol abilities and failures. Current Directions in Psychological Science, 21(5), 348-354.

Klopfer, F.J., \& Madden, T.M. (1980). The middlemost choice on attitude items: Ambivalence, neutrality, or uncertainty? Personality and Social Psychology Bulletin, 6(1), 97-101.

Lépine, R., Parrouillet, P., \& Camos, V. (2005). What makes working memory spans so predictive of high-level cognition? Psychonomic Bulletin \& Review, 12(1), 165170 .

Levinson, D.B., Smallwood, J., \& Davidson, R.J. (2012). The persistence of thought: evidence for a role of working memory in the maintenance of task-unrelated thinking. Psychological Science, 23(4), 375-380.

Luciana, M., Conklin, H.M., Hooper, C.J., \& Yarger, R.S. (2005). The development of nonverbal working memory and executive control processes in adolescents. Child Development, 76(3), 697-712.

Luck, S.J., \& Vogel, E.K. (1997). The capacity of visual working memory for features and conjunctions. Nature, 390(6657), 279-281.

Markman, E.M. (1979). Realizing that you don't understand: Elementary school children's awareness of inconsistencies. Child Development, 643-655.

Melby-Lervåg, M., Redick, T.S., \& Hulme, C. (2016). Working memory training does not improve performance on measures of intelligence or other measures of "far transfer" evidence from a meta-analytic review. Perspectives on Psychological Science, 11(4), 512-534.

Mills, C.M., \& Keil, F.C. (2004). Knowing the limits of one's understanding: The development of an awareness of an illusion of explanatory depth. Journal of Experimental Child Psychology, 87(1), 1-32.

Noles, N.S., Scholl, B.J., \& Mitroff, S.R. (2005). The persistence of object file representations. Perception \& Psychophysics, 67(2), 324-334.

Oberauer, K., \& Eichenberger, S. (2013). Visual working memory declines when more features must be remembered for each object. Memory \& Cognition, 41(8), 12121227.

Rademaker, R.L., Tredway, C.H., \& Tong, F. (2012). Introspective judgments predict the precision and likelihood of successful maintenance of visual working memory. Journal of Vision, 12(13), 21-21. 
Ricker, T.J. (2015). The role of short-term consolidation in memory persistence. AIMS Neuroscience, 2(4), 259-279.

Ricker, T.J., \& Cowan, N. (2014). Differences between presentation methods in working memory procedures: A matter of working memory consolidation. Journal of Experimental Psychology: Learning, Memory, and Cognition, 40(2), 417-428.

Ricker, T.J., \& Hardman, K.O. (2017). The nature of short-term consolidation in visual working memory. Journal of Experimental Psychology: General, 146(11), 15511573 .

Rouder, J.N., Morey, R.D., Cowan, N., Zwilling, C.E., Morey, C.C., \& Pratte, M.S. (2008). An assessment of fixed-capacity models of visual working memory. Proceedings of the National Academy of Sciences, 105(16), 5975-5979.

Salthouse, T.A. (2015). Do cognitive interventions alter the rate of age-related cognitive change? Intelligence, 53, 86-91.

Saults, J.S., \& Cowan, N. (2007). A central capacity limit to the simultaneous storage of visual and auditory arrays in working memory. Journal of Experimental Psychology: General, 136(4), 663-684.

Thiele, J.E., Pratte, M.S., \& Rouder, J.N. (2011). On perfect working-memory performance with large numbers of items. Psychonomic Bulletin \& Review, 18(5), 958-963.

Towse, J.N., Hitch, G.J., \& Hutton, U. (1998). A reevaluation of working memory capacity in children. Journal of Memory and Language, 39(2), 195-217.

Unsworth, N., Fukuda, K., Awh, E., \& Vogel, E.K. (2014). Working memory and fluid intelligence: Capacity, attention control, and secondary memory retrieval. Cognitive Psychology, 71, 1-26.

van den Berg, R., Awh, E., \& Ma, W.J. (2014). Factorial comparison of working memory models. Psychological Review, 121(1), 124-149.

Vergauwe, E., Barrouillet, P., \& Camos, V. (2010). Do mental processes share a domaingeneral resource? Psychological Science, 21(3), 384-390.

Wheeler, M.E., \& Treisman, A.M. (2002). Binding in short-term visual memory. Journal of Experimental Psychology: General, 131(1), 48-64.

Wilken, P., \& Ma, W.J. (2004). A detection theory account of change detection. Journal of Vision, 4(12): 11, 1120-1135. 
Wixted, J.T., \& Stretch, V. (2004). In defense of the signal detection interpretation of remember/know judgments. Psychonomic Bulletin \& Review, 11(4), 616-641.

Xenidou-Dervou, I., De Smedt, B., van der Schoot, M., \& van Lieshout, E.C. (2013). Individual differences in kindergarten math achievement: The integrative roles of approximation skills and working memory. Learning and Individual Differences, $28,119-129$.

Yeung, N., Botvinick, M.M., \& Cohen, J.D. (2004). The neural basis of error detection: conflict monitoring and the error-related negativity. Psychological Review, 111(4), 931-959.

Zhang, W., \& Luck, S.J. (2008). Discrete fixed-resolution representations in visual working memory. Nature, 453(7192), 233-235.

Zosh, J.M., \& Feigenson, L. (2012). Memory load affects object individuation in 18month-old infants. Journal of Experimental Child Psychology, 113(3), 322-336.

Zosh, J.M., \& Feigenson, L. (2015). Array heterogeneity prevents catastrophic forgetting in infants. Cognition, 136, 365-380. 
Christopher L. Blume began his academic career as an undergraduate student at the University of Missouri-Columbia. During this time he worked as an undergraduate research assistant in the Social Cognitive Neuroscience laboratory of Dr. Bruce Bartholow, the Cognitive Aging laboratory of Dr. Moshe Naveh-Benjamin, and the Working Memory laboratory of Dr. Nelson Cowan. Additionally during this time, Dr. Blume spent one semester studying at the National University of Ireland-Galway. Upon receiving his Bachelor of Arts Dr. Blume began working as a research assistant in both the Cognitive Aging lab and Working Memory labs mentioned above.

Dr. Blume's graduate career began by attending Georgia Southern University working in the Cognitive Psychology laboratory of Larry Locker. Dr. Blume completed a Master of Science degree with a thesis entitled "Functional Processing Aspects of Working Memory: Capacity Limitations and Mechanisms of Forgetting.” Dr. Blume followed this by returning to the University of Missouri-Columbia to work toward a PhD under the advisement of Dr. Nelson Cowan. Following almost one year of research Dr. Blume completed a Master of Arts degree with a thesis entitled "Repetition Effects in Object Switch Costs: Against a Switch Cost Measure of a Discrete Focus of Attention.” Over the next several years acted as instructor for multiple sections of the course “Learning, Memory, \& Cognition”, worked as a teaching assistant in ten separate courses, and worked on multiple research projects ranging from cognitive and developmental work on visual working memory, binding, and verbal working memory publishing in multiple academic psychology journals. Dr. Blume’s graduate career culminated with the current dissertation entitled "School-Aged Children's Awareness of their own Working Memory Contents.” 